\title{
Discrete Multitone Modulation With Principal Component Filter Banks
}

\author{
P. P. Vaidyanathan, Fellow, IEEE, Yuan-Pei Lin, Member, IEEE, Sony Akkarakaran, Member, IEEE, and \\ See-May Phoong, Associate Member, IEEE
}

\begin{abstract}
Discrete multitone (DMT) modulation is an attractive method for communication over a nonflat channel with possibly colored noise. The uniform discrete Fourier transforn (DFT) filter bank and cosine modulated filter bank have in the past been used in this system because of low complexity. We show in this paper that principal component filter banks (PCFB) which are known to be optimal for data compression and denoising applications, are also optimal for a number of criteria in DMT modulation communication. For example, the PCFB of the effective channel noise power spectrum (noise psd weighted by the inverse of the channel gain) is optimal for DMT modulation in the sense of maximizing bit rate for fixed power and error probabilities. We also establish an optimality property of the PCFB when scalar prefilters and postfilters are used around the channel. The difference between the PCFB and a traditional filter bank such as the brickwall filter bank or DFT filter bank is significant for effective power spectra which depart considerably from monotonicity. The twisted pair channel with its bridged taps, next and fext noises, and AM interference, therefore appears to be a good candidate for the application of a PCFB. This will be demonstrated with the help of numerical results for the case of the ADSL channel.
\end{abstract}

Index Terms-Channel capacity, digital subscriber loops (DSL), discrete multitone (DMT) modulation , frequency division multiplexing (FDM), principal component filter banks (PCFB).

\section{INTRODUCTION}

D ISCRETE multitone (DMT) modulation for nonflat channels has been studied by a number of authors in the last decade. The theoretical advantages of multitone modulation were demonstrated in the pioneering paper by Kalet [15] more than ten years ago. DMT has been considered seriously for use in digital subscriber loops (DSL), and excellent descriptions of this can be found in [10] and [31], The DMT system can be regarded as a filter bank in transmultiplexer configuration [1], [36], [41]. Typically, the filter banks used for this purpose are

Manuscript received December 11, 2001; revised April 3, 2002. This work was supported in part by the National Science Foundation under Grant MIP0703755, in part by the Office of Naval Research under Grant N00014-99-11002, in part by Microsoft Research, Redmond, WA, and in part by the National Science Council (NSC), Taiwan, R.O.C., under Grant 89-2213-E-009-118 and Grant 89-2213-E-002-122. This paper was recommended by Associate Editor W.-S. Lu.

P. P. Vaidyanathan and S. Akkarakaran are with the Department of Electrical Engineering, California Institute of Technology, Pasadena, CA 91125 USA (e-mail: ppvnath@sys.caltech.edu).

Y.-P. Lin is with the Department of Electrical and Control Engineering, National Chiao Tung University, Hsinchu, Taiwan, R.O.C.

S.-M. Phoong is with the Department of Electrical Engineering and Graduate Institute of Communication Engineering, National Taiwan University, Taipei, Taiwan, R.O.C.

Digital Object Identifier 10.1109/TCSI.2002.803249.
DFT filter banks which can be implemented efficiently with the FFT. The filters in these DFT filter banks provide poor separation between adjacent subchannels [27]. It is known that the use of better filters improves performance (e.g., higher bit rate for fixed error probabilities and power). This was clearly demonstrated in [27] using cosine modulated filter banks (CMFB) with sharp filters. Advantages of more general filter banks for this application are also described in [9]. In this paper, we consider a special type of orthonormal filter banks called the principal component filter bank (PCFB) (reviewed in Section IV) and show that it is optimal for the DMT application in a well-defined theoretical sense.

The filter responses in the PCFB depend on the channel transfer function and the noise power spectrum. Moreover even though PCFBs can be defined for infinite filter orders, they are evidently unrealizable. However, there appear to be two reasons why the optimality of the PCFB is of interest. First, it serves as a benchmark for comparing the performance of conventional unoptimized DMT systems which use the DFT, CMFB, and so forth. Second, in applications where the channel characteristics are fixed (e.g., twisted pair lines with standard next and fext noise) we can design the PCFB apriori and approximate it with practical digital filters. Such approximations can yield better performance than unoptimized designs like the DFT at the expense of higher complexity of implementation.

\section{A. Outline and Relation to Past Work}

The PCFB was introduced first in [32] and its optimality for a variety of problems was suggested in [35]. It has since been proved to be optimal for a general class of objective functions in signal processing [4], [24], [37], [46]. The role of a specific class of PCFBs in the optimality of DMT systems was first observed in [22]. A related problem, namely the optimization of filter bank precoders [13], [44] has been considered in great depth in a series of recent papers by Giannakis and his group [13], [28], [29]. The precoder typically introduces redundancy (like a non maximally decimated filter bank) to combat intersymbol interference. The precoder and receiver filters can be optimized according to several possible criteria. In this context, an excellent unification of several filter-bank based communication systems (including DMT) can be found in [28]. In this paper, we consider the specific role of the PCFB in the design of optimal orthonormal DMT systems. We believe this provides a fundamentally different viewpoint.

Two other excellent papers on related optimizations should be mentioned here. In [7], the authors consider many fundamental 
questions pertaining to transmission of signals in blocks, over dispersive channels. One of the results there is on the optimization of the covariance matrix of the transmitted block to maximize mutual information. The authors also show how such covariance can be realized by filter design. Next, a very general problem of filter bank optimization is handled in [30] where the authors optimize mutual information by optimizing the transmit and receive filters. Both zero forcing equalizers and minimum mean square equalizers come out of this elegant approach as special cases. In our paper, we consider the case of orthogonal filter banks with the perfect reconstruction property and assume that the channel is equalized by a zero forcing equalizer. Furthermore, we do not consider the mutual information but instead consider the optimization of useful quantities such as, for example, the actual bit rate with fixed error probabilities and transmitted power. This makes the problem much simpler and leads to very elegant insights. For example, it becomes clear that PCFBs optimize the bit rate for a fixed set of error probabilities and power. There is some commonality between the theme of our paper and the results in [30] and [7]. We shall see however, that the approach here is simpler and insightful because we focus directly on the PCFB solution based on simple convexity arguments.

In Section II, we describe the DMT system using multirate filter bank language and formulate a noise model. The benefits of optimizing the transmitting filters is motivated with a simple example in Section III. A brief review of PCFBs and their optimality is given in Section IV. More details on this section can be found in [4], [5]. Various criteria for the optimization of orthonormal DMT filter banks are presented in Section V and solutions presented. The role of principal component filter banks for asymmetric DSL (ADSL) service on twisted pairs is explained in Section VI, along with some numerical examples. Prefiltered orthonormal DMT systems (which are biorthogonal rather than orthonormal) are considered in Section VII and it is shown again that the PCFB has a role in optimality. Some parts of this paper have appeared in [38] and [39].

\section{B. DSP and Multirate Notations}

Bold faced letters denote matrices and vectors. The transpose, conjugate, and transpose-conjugate of a matrix are denoted, respectively, as $\mathbf{A}^{T}, \mathbf{A}^{*}$, and $\mathbf{A}^{\dagger}$. We use a subscript $c$ [e.g., $x_{c}(t), S_{c}(f)$ etc.] to distinguish continuous-time quantities from discretized versions. In general, the filters are allowed to be ideal (e.g., brickwall lowpass, etc.). So the $z$ transforms may not exist in any region of the $z$ plane. The notation $H(z)$ should be regarded as an abbreviation for the $\ell_{2}$ Fourier transform $H\left(e^{j \omega}\right)$. The language of multirate signal processing [36] will be used extensively throughout this paper. A summary of the most common ones follows.

1) The building block $\downarrow M$ in the figures denotes a decimator with input/output relation $y(n)=x(M n)$. The building block $\uparrow M$ denotes an expander with input/output relation

$$
y(n)= \begin{cases}x(n / M), & n=\text { multiple of } M \\ 0, & \text { otherwise. }\end{cases}
$$

The expander followed by a filter yields an interpolated version of $x(n)$. We use the notations $[x(n)]_{\downarrow M}$ and $[X(z)]_{\downarrow M}$ to denote the decimated version $x(M n)$ and its $z$-transform. Similarly, the expanded version is denoted by $[x(n)]_{\uparrow M}$, and its $z$-transform $X\left(z^{M}\right)$ by $[X(z)]_{\uparrow M}$. It can be shown that the Fourier transform of $x(M n)$ is a superposition of $X\left(e^{j \omega / M}\right)$ and $M-1$ shifted versions [36]

$$
\left[X\left(e^{j \omega}\right)\right]_{\downarrow M}=\frac{1}{M} \sum_{m=0}^{M-1} X\left(e^{j(\omega-2 \pi m) / M}\right) .
$$

2) Some standard abbreviations: a) PCFB: principal component filter bank, b) DMT: discrete multitone modulation, c) DSL: digital subscriber loop, d) ADSL: asymmetric DSL, e) PR: perfect reconstruction, f) KLT: Karhunen Loeve Transform, g) psd: power spectral density or power spectrum.

\section{DMT FILTER BANK}

Fig. 1(a) shows the essentials of DMT communication as required for the discussions of this paper. The signals $x_{k}(n)$ are $b_{k}$-bit symbols obtained from a PAM or QAM constellation (Appendix A). These symbols are interpolated $M$-fold by the filters $F_{k}(z)$ to obtain the subchannel or subband signals $u_{k}(n)$. The $k$ th transmitting filter has output

$$
u_{k}(n)=\sum_{i=-\infty}^{\infty} x_{k}(i) f_{k}(n-i M) .
$$

Fig. 1(b) demonstrates how this construction is done for the Oth filter $F_{0}(z)$, assumed to be lowpass. Essentially, we draw one copy of the impulse response sequence $f_{0}($.$) around every$ sample of $x_{0}(n)$ (separated by $M$ ) and add them up. The outputs of the filters $F_{1}(z), F_{2}(z)$ and so forth, are more complicated waveforms because they are bandpass. The filters $\left\{F_{k}\left(e^{j \omega}\right)\right\}$ traditionally cover different uniform regions of frequency as shown in Fig. 1(c). The signals $u_{k}(n)$ are analogous to modulated versions of the "baseband" sequence $x_{k}(n)$ because the bandwidth is shifted to the passband of $F_{k}(z)$. These are packed into $M$ adjacent frequency bands (passbands of the filters) and added to obtain the composite signal $x(n)$. Thus

$$
x(n)=\sum_{k=0}^{M-1} \sum_{i=-\infty}^{\infty} x_{k}(i) f_{k}(n-i M) .
$$

This signal is then sent through the channel which is represented by a transfer function $C(z)$ and additive Gaussian noise $e(n)$ with power spectrum $S_{e e}\left(e^{j \omega}\right)$. The received signal $y(n)$ is a distorted and noisy version of $x(n)$. The receiving filter bank $\left\{H_{k}(z)\right\}$ separates this signal into the components $y_{k}(n)$ which are distorted and noisy versions of the symbols $x_{k}(n)$. The task at this point is to detect the value of $x_{k}(n)$ from $y_{k}(n)$ with acceptable error probability.

In actual practice, the channel is a continuous-time system $C_{c}(s)$ preceded by $D / A$ conversion and followed by $A / D$ conversion. We have replaced this with discrete equivalents $C(z)$ and $e(n)$. The original motivation behind multitone modulation [15] is that the power and/or bits could be allocated in an efficient manner in the subchannels, depending on the channel gain 


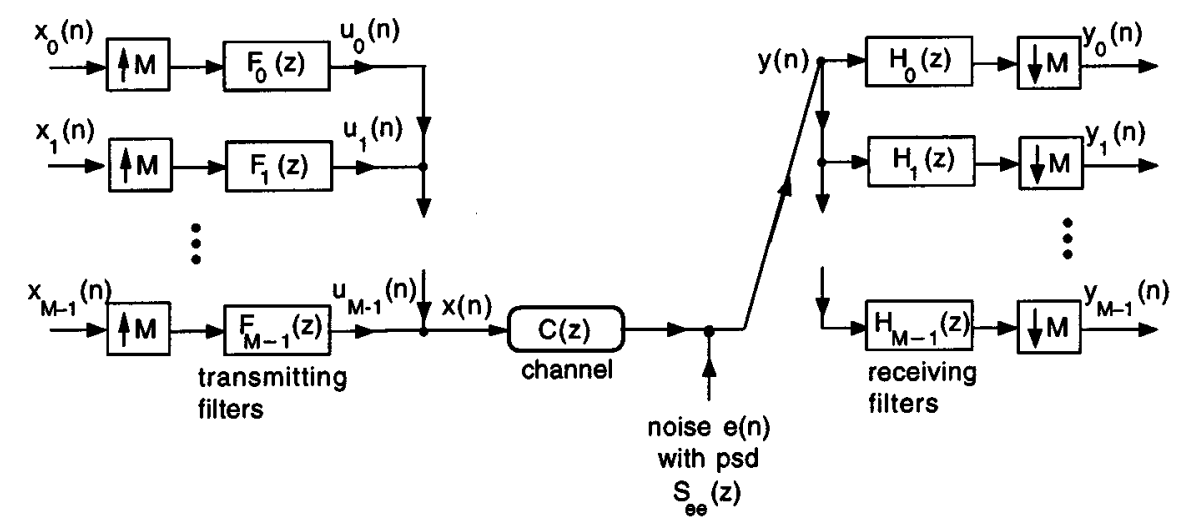

(a)

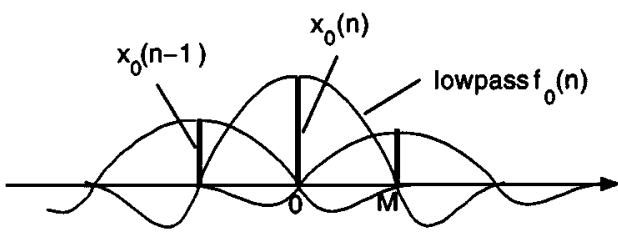

(b)

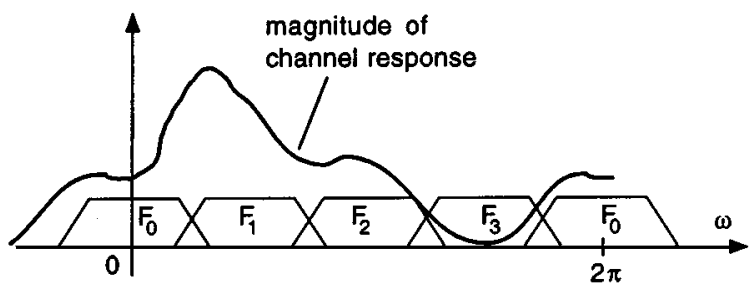

(c)

Fig. 1. (a) The DMT communication system. (b) The interpolation or modulation performed by the transmitting filter $F_{0}(z)$. (c) An example of responses of the transmitting filters.

$\left|C\left(e^{j \omega}\right)\right|^{2}$, and noise psd $S_{e e}\left(e^{j \omega}\right)$ in that subchannel. In this way, the classical water filling idea for resource allocation [11], [26] could be approximated. For a given transmitted power and probability of error, this yields a better bit rate than direct single tone modulation (assuming no channel coding). The DMT idea is similar in principle to subband coding where a signal $x(n)$ to be quantized is first decomposed into subbands. Background material on the DMT system and more generally on the use of digital filter banks in communications can be found in [1], [10], [15], [17], [20], [33].

\section{A. Perfect Reconstruction}

In absence of the channel noise $e(n)$, the DMT system of Fig. 1(a) is LTI, with the transfer function from $x_{m}($.$) to y_{k}($. given by

$$
D_{k m}(z)=\left.H_{k}(z) C(z) F_{m}(z)\right|_{\downarrow M} .
$$

In general, the symbol $y_{k}(n)$ is therefore affected by $x_{m}(i)$ when $m \neq k$, resulting in interband interference. For the case $k=m$, if the quantity $D_{k k}(z)$ is not a constant, then $y_{k}(n)$ is affected by $x_{k}(i)$ when $n \neq i$, and we have intraband interference. The condition to eliminate these two interferences is

$$
\left.H_{k}(z) C(z) F_{m}(z)\right|_{\downarrow M}=\delta(k-m) .
$$

If interband and intraband interferences are eliminated, the DMT system is said to be free from intersymbol interference (ISI). We then have the perfect reconstruction or PR property $y_{k}(n)=x_{k}(n)$ for all $k$ (in absence of noise). If the filter responses in Fig. 1(b) are nonoverlapping, then the subchannels are completely isolated. There is no interband ISI, though we might still have intraband ISI. Even if the filters have overlap as in any practical implementation, we can still avoid both types of ISI as long as (3) holds. In fact, the most popular DMT system uses a DFT filter bank where the filters have significant overlap even though (3) holds.

Biorthogonality: The filter bank $\left\{F_{m}, H_{k}\right\}$ is said to be biorthogonal if

$$
\left.H_{k}(z) F_{m}(z)\right|_{\downarrow M}=\delta(k-m) .
$$

This means that the impulse response $g_{k m}(n)$ of the product filter $H_{k}(z) F_{m}(z)$ has the Nyquist $(M)$ or zero-crossing property

$$
g_{k m}(M n)=0
$$

for $k \neq m$ and $g_{k k}(M n)=\delta(n)$. Under this biorthogonality condition, we have perfect reconstruction only if $C(z)=1$. In this paper, we shall make the simplifying assumption that $\left\{F_{m}, H_{k}\right\}$ is biorthogonal and that the channel transfer function $C(z)$ is equalized by using the inverse filter or zero-forcing equalizer $1 / C(z)$ just before entering the bank of filters $\left\{H_{k}(z)\right\}$. The path from $x_{k}(n)$ to $y_{k}(n)$ now has the effective form shown in Fig. 2(a). ${ }^{1}$ In actual practice, there are many ways to approximate this equalized system (see [25] and references therein). One approach would be to use a time domain equalizer in cascade with the channel and reduce the effective channel to be FIR with a short impulse response. This effective FIR filter is then compensated for by the use of a cyclic prefix followed by appropriate multipliers at the outputs of $H_{k}(z)$, called frequency domain equalizers.

\footnotetext{
${ }^{1}$ We make the assumption that $C\left(e^{j \omega}\right) \neq 0$ for any $\omega$. Otherwise, we have to leave out the offending frequency band.
} 


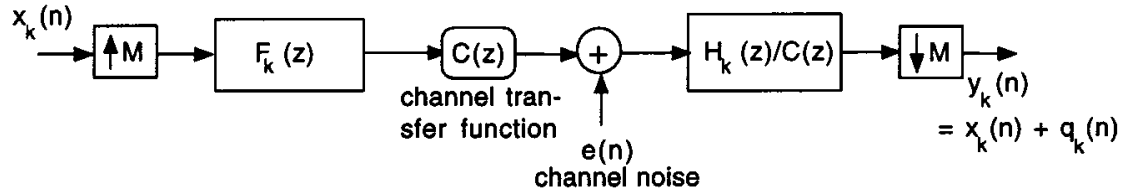

(a)

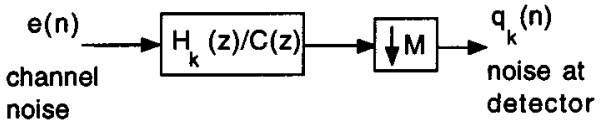

(b)

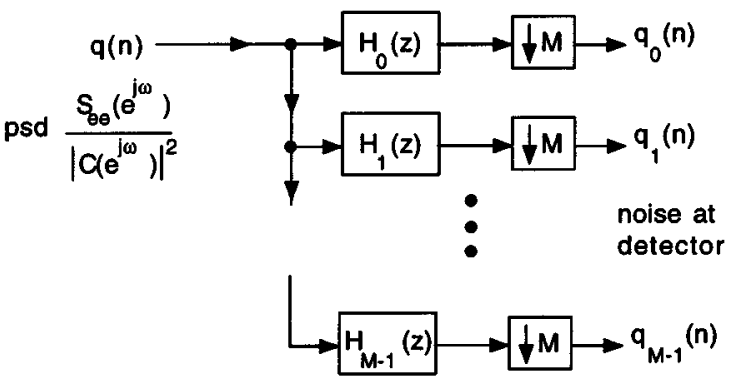

(c)

Fig. 2. (a) The path from $x_{k}(n)$ to $y_{k}(n)$ in a DMT system with ideal equalizer $1 / C(z)$. (b) Noise processing in the $k$ th subchannel. (c) Complete noise model.

This is explained at length in [10] for DFT based DMT, and a modification for general DMT has been advanced in [22].

\section{B. Channel Noise Model}

Now consider the effect of channel noise $e(n)$. Assuming that $\left\{F_{m}, H_{k}\right\}$ is biorthogonal and that $1 / C(z)$ is inserted as shown in Fig. 2(a), the $k$ th received symbol at time $n$ is given by

$$
y_{k}(n)=x_{k}(n)+q_{k}(n)
$$

where $q_{k}(n)$ is the channel noise filtered through $H_{k}(z) / C(z)$ and decimated [Fig. 2(b)]. If the channel noise is wide sense stationary with power spectrum $S_{e e}(z)$ then, the variance of $q_{k}(n)$ is

$$
\sigma_{q k}^{2}=\int_{0}^{2 \pi} \frac{S_{e e}\left(e^{j \omega}\right)}{\left|C\left(e^{j \omega}\right)\right|^{2}}\left|H_{k}\left(e^{j \omega}\right)\right|^{2} d \omega / 2 \pi .
$$

Notice that the noise $q_{k}(n)$ at the detector input can be viewed as the output of a maximally decimated analysis bank $\left\{H_{k}(z)\right\}$ in response to an effective noise source $q(n)$ with effective noise psd

$$
S_{q q}\left(e^{j \omega}\right)=\frac{S_{e e}\left(e^{j \omega}\right)}{\left|C\left(e^{j \omega}\right)\right|^{2}}
$$

This yields the noise model shown in Fig. 2(c).

Optimization of the DMT Filter Bank: We see that there is some control on the variances of $q_{k}(n)$, because we can choose the filters $\left\{H_{k}(z)\right\}$. We can take advantage of this. Even if we assume that the filters are allowed to be ideal, it turns out that the brickwall filter stacking shown in Fig. 1(c) is not necessarily the best choice (Section III). For any given channel, we can define a filter bank called the principal component filter bank. The frequency partitioning generated by such a filter bank is optimal for the channel (Section V).

\section{Probability of Error, Transmitted Power, and Bit Rate}

For simplicity, we assume that $x_{k}(n)$ are PAM symbols (Appendix $\mathrm{A})$. Assuming that $x_{k}(n)$ is a random variable with $2^{b_{k}}$ equiprobable levels, its variance represents the average power $P_{k}$ in the symbol $x_{k}(n)$. The Gaussian channel noise $e(n)$ is filtered through $H_{k}(z) / C(z)$ and decimated by $M$. For the purpose of variance calculation, the model for the noise $q_{k}(n)$ at the detector input can therefore be taken as in Fig. 2(c). Let $\sigma_{q_{k}}^{2}$ be the variance of $q_{k}(n)$. Then, the probability of error in detecting the symbol $x_{k}(n)$ is given by [26]

$$
\mathcal{P}_{e}(k)=2\left(1-2^{-b_{k}}\right) \mathcal{Q}\left(\sqrt{\frac{3 P_{k}}{\left(2^{2 b_{k}}-1\right) \sigma_{q_{k}}^{2}}}\right)
$$

where $\mathcal{Q}(v) \triangleq \int_{v}^{\infty} e^{-u^{2} / 2} d u / \sqrt{2 \pi}$ (area of the normalized Gaussian tail). Since the $\mathcal{Q}$-function can be inverted for any nonnegative argument, we can invert (8) to obtain

$$
P_{k}=\beta\left(\mathcal{P}_{e}(k), b_{k}\right) \times \sigma_{q_{k}}^{2}
$$

where the exact nature of the function $\beta(.,$.$) is not of immediate$ interest. This expression says that if the probability of error has to be $\mathcal{P}_{e}(k)$ or less at the bit rate $b_{k}$, then the power in $x_{k}(n)$ has to be at least as large as $P_{k}$. The required total transmitted power is therefore

$$
P=\sum_{k=0}^{M-1} P_{k}=\sum_{k=0}^{M-1} \beta\left(\mathcal{P}_{e}(k), b_{k}\right) \times \sigma_{q k}^{2} .
$$

Suppose $x(n)$ is converted into a continuous time signal $x_{c}(t)$ by the D/A converter at sampling rate $1 / T$ so that $x_{c}(n T)=$ $x(n)$. If a voltage waveform $x_{c}(t) \mathrm{V}$ is applied across a $1-\Omega$ resistor, the power delivered is actually $P \mathrm{~W}$, regardless of the sample spacing $T$. The samples of $x_{k}(n)$ are separated by $M T$ s. With $b_{k}$ representing the number of bits per sample in $x_{k}(n)$, the $k$ th subchannel therefore carries $b_{k} / M T$ bits/s. The total bit rate is therefore

$$
\frac{1}{M T} \sum_{k=0}^{M-1} b_{k} \quad \text { bits/s }
$$

or equivalently, $b / M T$ bits/s where $b \triangleq \sum b_{k}$. 


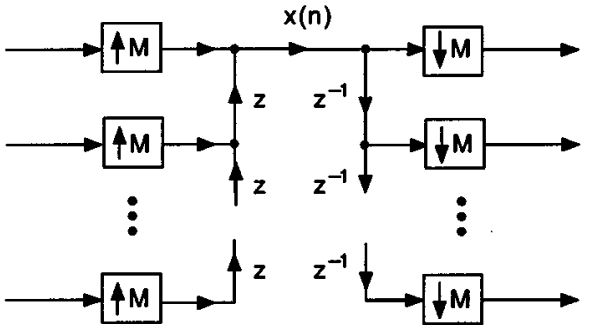

(a)

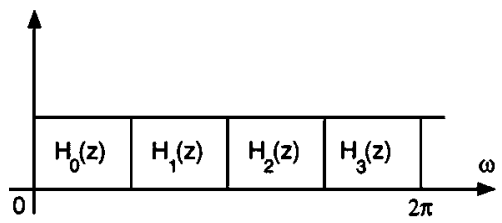

(b)

Fig. 3. Examples of orthonormal filter banks. (a) Delay chain system. (b) Brickwall filter bank with contiguous stacking $(M=4)$.

\section{Orthonormal DMT Systems}

The set of $M$ filters $\left\{H_{k}(z)\right\}$ is said to be orthonormal if

$$
\left.H_{k}^{*}\left(e^{j \omega}\right) H_{m}\left(e^{j \omega}\right)\right|_{\downarrow M}=\delta(k-m) .
$$

In an orthonormal DMT system we choose the transmit filters to be $F_{k}\left(e^{j \omega}\right)=H_{k}^{*}\left(e^{j \omega}\right)$, i.e., $f_{k}(n)=h_{k}^{*}(-n)$. This ensures biorthogonality (4), and furthermore the filters $\left\{F_{k}\right\}$ satisfy $\left.F_{k}^{*}\left(e^{j \omega}\right) F_{m}\left(e^{j \omega}\right)\right|_{\downarrow M}=\delta(k-m)$. In terms of impulse responses, this orthonormality condition is equivalent to

$$
\sum_{n} f_{k}(n-i M) f_{m}^{*}(n-\ell M)=\delta(k-m) \delta(i-\ell) .
$$

Thus, the composite signal $x(n)$ in (2) can be regarded as a superposition of elements from an orthonormal set. In fact, any subchannel signal $u_{k}(n)$ is a superposition of elements from the orthonormal set $\left\{f_{k}(n-i M)\right\}$ as seen from (1). Fig. 3 shows two extreme examples of orthonormal filter banks. The first one is the delay chain system $\left[H_{k}(z)=z^{-k}\right.$ and $\left.F_{k}(z)=z^{k}\right]$ and the second is the ideal brickwall filter bank.

For $k=m$, (11) yields $\left.\left|H_{k}\left(e^{j \omega}\right)\right|^{2}\right|_{\downarrow M}=1$. If the impulse response of $\left|H_{k}\left(e^{j \omega}\right)\right|^{2}$ is denoted as $g_{k}(n)$ then the preceding condition is equivalent to the $\operatorname{Nyquist}(M)$ or zero-crossing constraint $g_{k}(M n)=\delta(n)$. Similarly, for biorthogonal filter banks, the product $H_{k}(z) F_{k}(z)$ is $\operatorname{Nyquist}(M)$. Orthonormal filter banks have been thoroughly studied [36], [42]. Here are some of their properties: 1) $\int_{0}^{2 \pi}\left|H_{k}\left(e^{j \omega}\right)\right|^{2} d \omega / 2 \pi=1$ (unit energy property); 2) $\left|H_{k}\left(e^{j \omega}\right)\right|^{2} \leq M$ (boundedness); 3) $\sum_{k=0}^{M-1}\left|H_{k}\left(e^{j \omega}\right)\right|^{2}=M$ (power complementarity). Stated here for $\left\{H_{k}(z)\right\}$, these also hold for $\left\{F_{k}(z)\right\}$.

\section{E. Polyphase Representation of DMT Systems}

Using the polyphase notations described, for example, in [36, Ch. 5], we can express the row vector of transmitting filters and the column vector of receiving filters as

$$
\begin{aligned}
{\left[\begin{array}{llll}
F_{0}(z) & F_{1}(z) & \cdots & F_{M-1}(z)
\end{array}\right] } & =\mathbf{d}^{T}\left(z^{-1}\right) \mathbf{R}\left(z^{M}\right) \\
{\left[\begin{array}{llll}
H_{0}(z) & H_{1}(z) & \cdots & H_{M-1}(z)
\end{array}\right]^{T} } & =\mathbf{E}\left(z^{M}\right) \mathbf{d}(z)
\end{aligned}
$$

where $\mathbf{d}(z)$ is the delay chain vector defined by $\mathbf{d}(z)=$ $\left[\begin{array}{lllll}1 & z^{-1} & z^{-2} & \cdots & z^{-(M-1)}\end{array}\right]^{T}$. The DMT system can therefore be redrawn as in Fig. 4(a). Using Noble identities [36] the decimator and expander can be moved as shown in Fig. 4(b). This is the polyphase representation of the DMT filter bank. Note that the noise model shown in Fig. 2(c) can be redrawn in polyphase form as shown in Fig. 4(c). This will be quite insightful as we shall see.

The biorthogonality property (4) can be shown to be equivalent to $\mathbf{E}(z) \mathbf{R}(z)=\mathbf{I}$. The special case where the matrix $\mathbf{E}\left(e^{j \omega}\right)$ is unitary for all $\omega$ corresponds to orthonormal DMT systems. In this case, we choose $\mathbf{R}\left(e^{j \omega}\right)=\mathbf{E}^{\dagger}\left(e^{j \omega}\right)$ (transpose conjugate) for perfect reconstruction. The DMT systems where $\mathbf{R}(z)$ is a constant unitary matrix $\mathbf{T}$ has been of some practical importance. In this case, the filters $F_{k}(z)$ are FIR with lengths $\leq M$. This is the DMT counter part of the transform coder in subband coding theory. The example where $\mathbf{T}$ is the DFT matrix falls under this class.

\section{OPtiMAL CHOICE OF DMT FILTER BANK}

To motivate the usefulness of optimizing the transmitter and receiver filters, consider Fig. 5(a). This shows an example of the effective noise psd $S_{c}(f)$ in terms of the continuous-time frequency variable $f$. [The discretized version of this is $S_{q q}\left(e^{j \omega}\right)$ defined in (7)]. This is assumed bandlimited to $1 \mathrm{MHz}$. The units for $S_{c}(f)$ are in milliwatts per hertz, and a decibel plot would show $10 \log _{10} S_{c}(f)$ in $\mathrm{dB} \cdot \mathrm{m} / \mathrm{Hz}$ as in the figure. Using a sampling rate of $2 \mathrm{MHz}$, the digital version $S_{e e}\left(e^{j \omega}\right) /\left|C\left(e^{j \omega}\right)\right|^{2}$ of the psd $S_{c}(f)$ is as shown in Fig. 5(b) where $c=2 * 10^{-4}$ (due to the factor $1 / T$ in the Fourier transform after sampling). These are not unrealistic numbers for typical twisted pair telephone channels for which DMT modulation is the standard. The two bumps (each assumed 10-kHz wide) can be regarded as oversimplified versions of the effects of bridged taps (first bump) and AM noise (second bump) [31]. The rapid decay of channel gain is not depicted in this "toy" example, but we shall do that in Section VI. Consider a two-band DMT system $(M=2)$. One choice of the orthonormal filter bank, namely the brickwall stacking, is shown in Fig. 5(c). With the effective psd $S_{q q}\left(e^{j \omega}\right)$ as in Fig. 5(b) we can now calculate the variances $\sigma_{q k}^{2}$. Let us pick some values for the remaining parameters.

1) Error probabilities $\mathcal{P}_{e}(0)=\mathcal{P}_{e}(1)=10^{-9}$.

2) $b_{0}=6$ and $b_{1}=2$. These are the bits in the PAM constellations for $x_{0}(n)$ and $x_{1}(n)$. It makes sense to use smaller value for $b_{1}$ because there is more noise in the region covered by $H_{1}\left(e^{j \omega}\right)$. Since the average of $b_{k} \mathrm{~s}$ is 4 , the average bit rate for the 2-MHz sampling rate is $8 \mathrm{Mbits} / \mathrm{s}$.

The average power $P$ needed to meet these requirements can be calculated from (10), and the result turns out to be $56 \mathrm{~mW}$. Instead of using the brickwall filter bank suppose we use the filter bank shown in Fig. 5(d) and (e). We still have two subbands $(M=2)$ but each filter now has two passband regions. It can be verified that this filter bank still satisfies orthonormality (11). We can recalculate the variances $\sigma_{q k}^{2}$ now and compute the average power. The result is $5.67 \mathrm{~mW}$. Thus

savings in total power $=56 / 5.67 \approx 9.9$ 


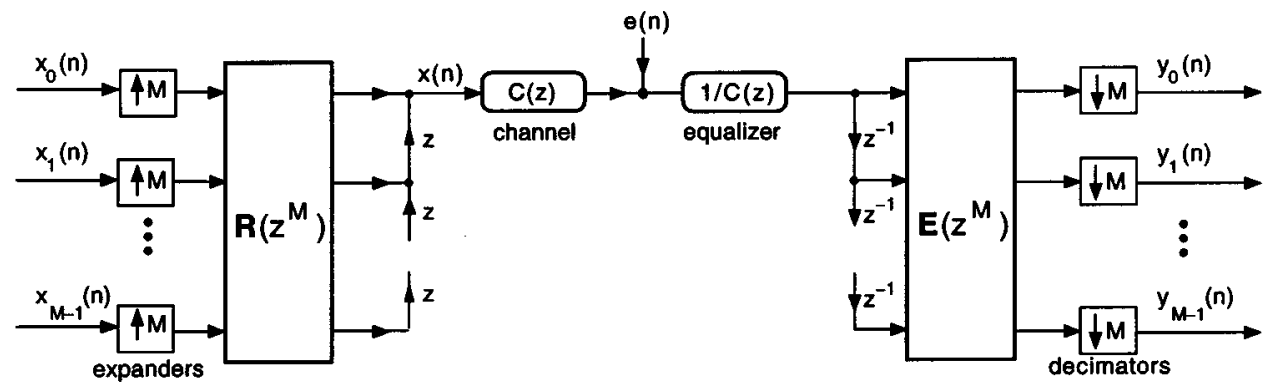

(a)

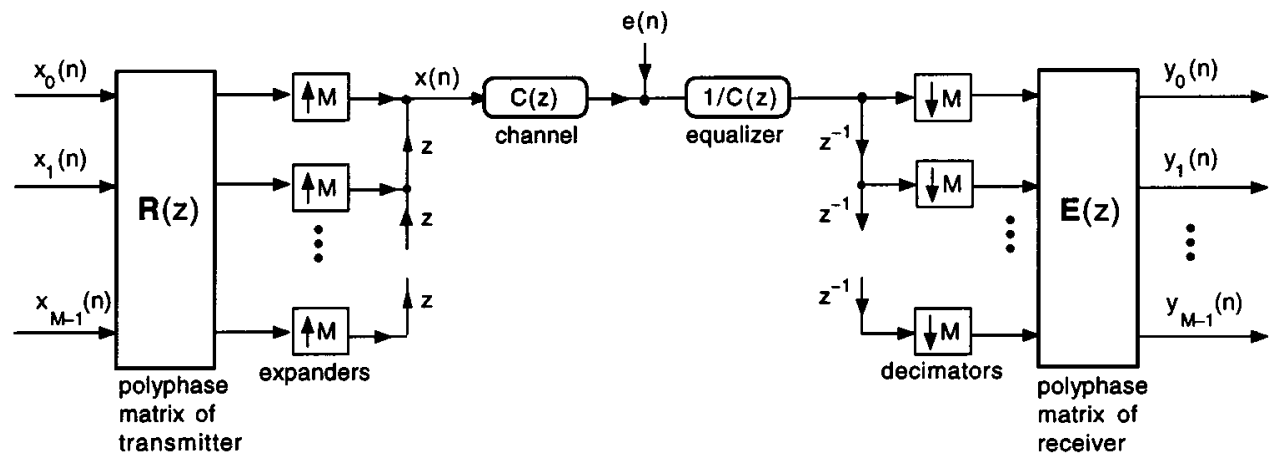

(b)

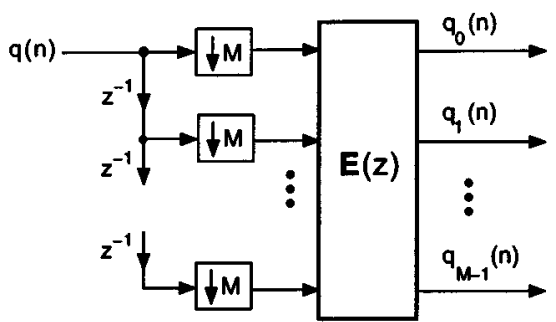

(c)

Fig. 4. (a) Polyphase representation of the DMT filter bank. (b) Simplification using multirate identities. (c) Noise model in polyphase form.

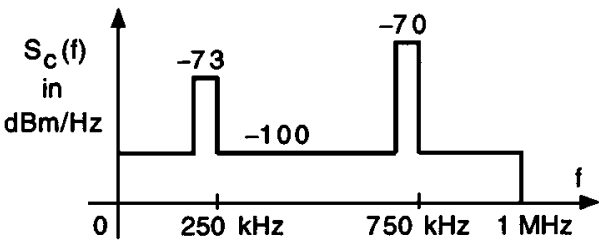

(a)

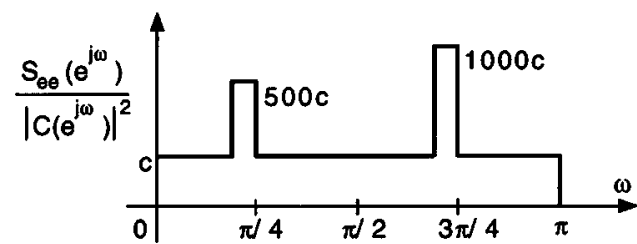

(b)

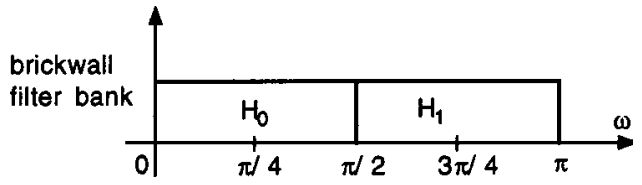

(c)

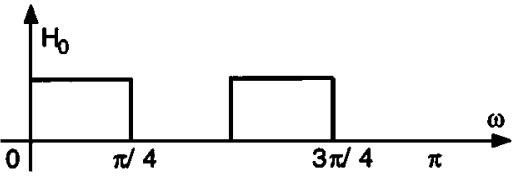

(d)

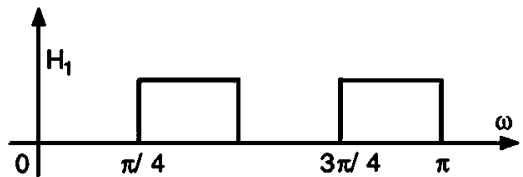

(e)

Fig. 5. Demonstrating the effectiveness of good choice of filter banks in the DMT system. (a) Effective noise psd. (b) Digitized version. (c) Traditional brickwall filter bank for $M=2$. (d) and (e) Different choice of filter bank.

or about $10 \mathrm{~dB}$. In summary, the modified filter bank achieves the bit rate of $8 \mathrm{Mbits} / \mathrm{s}$ and error probability of $10^{-9}$ using almost $10 \mathrm{~dB}$ less power!
The difference between the two filter banks in the example is that the variances $\sigma_{q_{k}}^{2}$ (whose sum is fixed by orthonormality) are distributed differently depending on the shape of the effec- 
tive noise psd $S_{q q}\left(e^{j \omega}\right)$. The natural question then is: given an effective noise psd and an arbitrary $M$, how do we choose the orthonormal filter bank $\left\{H_{k}\left(e^{j \omega}\right)\right\}$ to minimize the transmitted power for fixed specifications? The answer is that $\left\{H_{k}\left(e^{j \omega}\right)\right\}$ should be chosen as a principal component filter bank for the effective noise psd.

\section{PRINCIPAl COMPONENT FILTER BANKS}

To define a PCFB first consider two sets of $M$ nonnegative numbers $\left\{a_{n}\right\}$ and $\left\{b_{n}\right\}$. We say that $\left\{a_{n}\right\}$ majorizes $\left\{b_{n}\right\}$ if, after reordering such that $a_{n} \geq a_{n+1}$ and $b_{n} \geq b_{n+1}$, we have

$$
\sum_{n=0}^{P} a_{n} \geq \sum_{n=0}^{P} b_{n}
$$

for $0 \leq P \leq M-1$, with equality for $P=M-1$. Thus, all the partial sums in $\left\{a_{n}\right\}$ dominate those in $\left\{b_{n}\right\}$. Consider a given class $\mathcal{C}$ of $M$-band uniform orthonormal filter banks. This class can be the class $\mathcal{C}_{t c}$ of transform coders (with filter lengths $\leq M$ ), or the class $\mathcal{C}_{\text {ideal }}$ of ideal filter banks (filters allowed to have infinite order, like brickwall filters). Or it could be a practically attractive class like the FIR class $\mathcal{C}_{\text {fir }}$ with filter orders bounded by a fixed integer, or the so-called cosine modulated class $\mathcal{C}_{c m f b}$ [36]. Given such a class $\mathcal{C}$ and an input power spectrum $S_{q q}\left(e^{j \omega}\right)$ we say that a filter bank $\mathcal{F}$ in $\mathcal{C}$ is a principal component filter bank or PCFB if the set $\left\{a_{k}\right\}$ of its subband variances [i.e., variances $\sigma_{q_{k}}^{2}$ of the signals $q_{k}(n)$ in Fig. 2(c)] majorizes the set $\left\{b_{k}\right\}$ of subband variances of all other filter banks in the class. That is, with $a_{n} \geq a_{n+1}$ and $b_{n} \geq b_{n+1}$,

$$
a_{0} \geq b_{0}, \quad a_{0}+a_{1} \geq b_{0}+b_{1}, \quad \ldots
$$

and so forth. The equality $\sum_{k=0}^{M-1} a_{k}=\sum_{k=0}^{M-1} b_{k}$ follows automatically from orthonormality.

The advantage of PCFBs is that they are optimal for several problems. This includes subband coding with arbitrary (not necessarily high) bit rates, the denoising problem, and so forth, as elaborated in [4]. These arise from the result (proved in [4]) that any concave function $\phi$ of the subband variance vector

$$
\mathbf{v}=\left[\begin{array}{llll}
\sigma_{q_{0}}^{2} & \sigma_{q_{1}}^{2} & \cdots & \sigma_{q_{M-1}}^{2}
\end{array}\right]^{T}
$$

is minimized by a PCFB when one exists. Similarly any convex function is maximized by a PCFB. Note that any permutation of the filters in a PCFB retains the PCFB property. Thus, given a particular (concave or convex) objective, we have to choose the right permutation so that the objective is optimized.

Using the preceding results we show in this paper, that PCFBs also serve as optimal solutions to certain problems in communication systems which use DMT modulation. It is possible that PCFBs do not exist for certain classes (e.g., see [4]). But when they exist, they have the stated optimality. Whenever we say that the PCFB is optimal for a problem, the implicit assumption is that the class of filter banks searched is such that a PCFB exists.

\section{A. Construction of the PCFB, and Compaction Filters}

For the transform coder class $\mathcal{C}_{t c}$ the filters have length $\leq M$. This means that the polyphase matrix $\mathbf{E}(z)$ in Fig. 4(c) is a constant matrix $\mathbf{T}$. Suppose $\mathbf{R}_{q q}$ denotes the $M \times M$ autocorre- lation matrix of its input vector. If $\mathbf{T}$ is chosen as the unitary matrix diagonalizing $\mathbf{R}_{q q}$ then, it defines the PCFB in this case. This $\mathbf{T}$ is nothing but the Karhunen-Loeve transform (KLT) of the effective noise input $q(n)$. This choice decorrelates the signals $q_{k}(n)$ for each $n$. That is, the autocorrelation matrix of the vector

$$
\left[\begin{array}{llll}
q_{0}(n) & q_{1}(n) & \cdots & q_{M-1}(n)
\end{array}\right]^{T}
$$

is diagonalized. For the ideal filter bank class $\mathcal{C}_{\text {ideal }}$, the matrix $\mathbf{E}(z)$ could have infinite order in $z$. This means in particular that ideal filters $H_{k}\left(e^{j \omega}\right)$ are allowed. In this case, the PCFB is such that the power spectrum of the vector (12) is diagonalized which in particular means that the autocorrelation matrix is diagonal as well. In short, the KLT forces the instantaneous decorrelation property $E\left[q_{k}(n) q_{m}^{*}(n)\right]=0$ for each $n$, whereas the PCFB for $\mathcal{C}_{\text {ideal }}$ forces the total decorrelation property $E\left[q_{k}(n) q_{m}^{*}(i)\right]=0$ for all $n, i$ (with $k \neq m$ ). In addition, the PCFB for $\mathcal{C}_{\text {ideal }}$ also induces the spectral majorization property. That is, assuming $\sigma_{q_{i}}^{2}$ are in decreasing order, the power spectra $S_{i}\left(e^{j \omega}\right)$ of $q_{i}(n)$ are ordered such that $S_{i}\left(e^{j \omega}\right) \geq S_{i+1}\left(e^{j \omega}\right)$ pointwise for all $\omega$. It has been shown in [37] that total decorrelation and spectral majorization are necessary and sufficient for the PCFB property in the class $\mathcal{C}_{\text {ideal }}$. For classes other than $\mathcal{C}_{\text {ideal }}$ and the transform coder class, such conditions for the PCFB property have not been established. In fact, the existence of a PCFB is not guaranteed for arbitrary classes of orthonormal filter banks (see [4] for counterexample). When a PCFB does exist, there is a sequential algorithm that can be used to construct the filters [23], [4], [5].

Closely associated with PCFBs is the notion of an optimal compaction filter $H\left(e^{j \omega}\right)$ for a signal $q(n)$ with power spectrum $S_{q q}\left(e^{j \omega}\right)$. Such a filter has the property that its output in response to the input $q(n)$ has maximum variance subject to the Nyquist $(M)$ constraint $\left.\left|H\left(e^{j \omega}\right)\right|^{2}\right|_{\downarrow M}=1$. For the transform coder class, this filter can be constructed by making $\mathbf{E}(z)$ the KLT, and taking the receiver filter with largest variance as the solution. For the class $\mathcal{C}_{\text {ideal }}$ the optimal compaction filter can be constructed graphically [37]. Typically, there are multiple passbands. For example, the power spectrum in Fig. 6(a) has optimal compaction filter for $M=4$ shown in Fig. 6(b). To construct such a filter we proceed as follows: take any frequency $\omega_{0}$ in $0 \leq \omega_{0}<2 \pi / M$ and consider the set of $M$ frequencies

$$
\omega_{0}+\frac{2 \pi m}{M}, \quad 0 \leq m \leq M-1
$$

Choose one frequency in this set such that $S_{q q}\left(e^{j \omega}\right)$ is a maximum in this set (if there are multiple maxima choose one arbitrarily). Include this frequency in the passband of $H\left(e^{j \omega}\right)$, and the remaining $M-1$ frequencies in the stopband. Repeat this for all $\omega_{0}$ in $0 \leq \omega_{0}<2 \pi / M$. Set the passband height equal to $\sqrt{M}$ and stopband height equal to zero. This completely determines the optimal compaction filter $H\left(e^{j \omega}\right)$ for the power spectrum $S_{q q}\left(e^{j \omega}\right)$.

The PCFB can be constructed by designing the filters $H_{0}\left(e^{j \omega}\right), H_{1}\left(e^{j \omega}\right), \ldots$ sequentially one at a time as follows [37]. First, design $H_{0}\left(e^{j \omega}\right)$ as an optimal compaction filter for $S_{q q}\left(e^{j \omega}\right)$. Then, define a partial power spectrum by removing or 


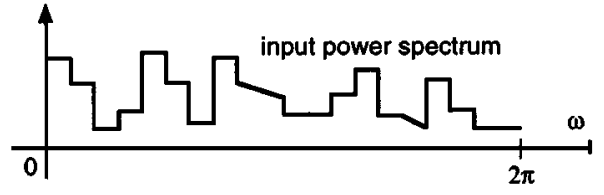

(a)

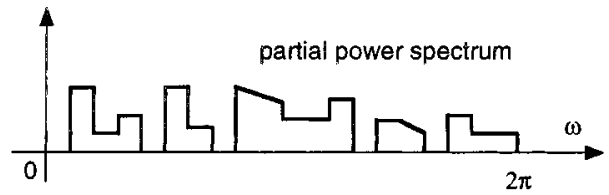

(c)

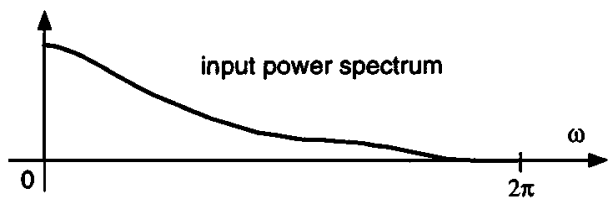

(e)

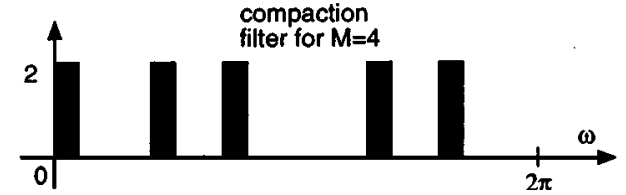

(b)

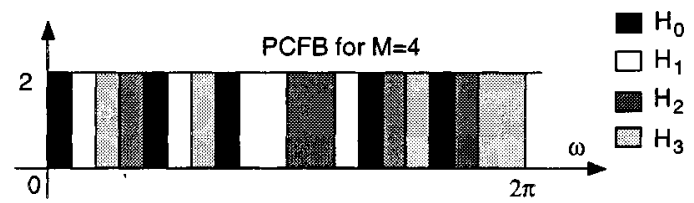

(d)

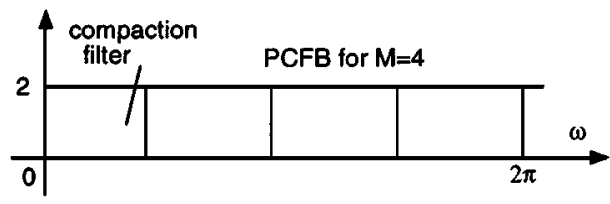

(f)

Fig. 6. (a) A power spectrum. (b) Optimum compaction filter for $M=4$ for (a). (c) Partial power spectrum obtained by peeling. (d) PCFB for $M=4$. (e) A monotone power spectrum. (f) PCFB for $M=4$ for (e).

peeling off from $S_{q q}\left(e^{j \omega}\right)$ those parts that fall in the passbands of $H_{0}\left(e^{j \omega}\right)$ [Fig. 6(c)]. Design an optimal compaction filter $H_{1}\left(e^{j \omega}\right)$ for this partial psd. Remove those parts of this partial psd that fall under the passband of $H_{1}\left(e^{j \omega}\right)$, and continue this until all the filters have been designed. Fig. 6(d) shows the filters designed in this manner for $M=4$.

Filter banks constructed using this procedure have the following properties.

1) A filter $H_{k}\left(e^{j \omega}\right)$ may have more than one passband, but the sum of all its passband widths is equal to $2 \pi / M$, and the heights of the passbands are equal to $\sqrt{M}$ (e.g., two in Fig. 6).

2) The passbands of any two filters are disjoint, and the filters together cover the entire frequency range.

3) For any $\omega_{0}$ consider the set of $M$ frequencies in (13). Given any filter $H_{k}\left(e^{j \omega}\right)$, exactly one of these $M$ frequencies belongs to its passband, and the others belong to the stopband. This property implies two things: a) Each filter is an aliasfree $(M)$ filter. In other words, its output can be decimated by $M$ without causing overlaps of the copies of the spectrum created by downsampling; b) The decimated version $\left|H_{k}\left(e^{j \omega}\right)\right|_{\downarrow M}^{2}=1$ for all $\omega$.

It readily follows from these that the resulting filter bank is orthonormal. The proof that this is actually a PCFB can be found in [37]. For the case of a monotone decreasing power spectrum $S_{q q}\left(e^{j \omega}\right)$ the compaction filter is lowpass, as demonstrated in Fig. 6(e) and (f). In this case, the PCFB happens to be the traditional brickwall stacking of bandpass filters as shown.

\section{OPTIMIZATION OF THE DMT FILTER BANK}

In this section, we show how to optimize the orthonormal filter bank used in a DMT system. We assume that the number of subchannels $M$ is fixed. The channel transfer function $C(z)$ and the noise power spectrum $S_{e e}\left(e^{j \omega}\right)$ are assumed to be fixed and known as well. A brief overview of these results will also appear in [40].

\section{A. Minimizing Transmitted Power}

Recall that the total transmitted power can be expressed in terms of the error probability and noise variances as shown in (10)

$$
P=\sum_{k=0}^{M-1} P_{k}=\sum_{k=0}^{M-1} \beta\left(\mathcal{P}_{e}(k), b_{k}\right) \times \sigma_{q k}^{2}
$$

Let us assume that the bit rates $b_{k}$ and probabilities of error $\mathcal{P}_{e}(k)$ are fixed. For this desired combination of $\left\{b_{k}\right\}$ and $\left\{\mathcal{P}_{e}(k)\right\}$, the total power required depends on the distribution of noise variances $\left\{\sigma_{q_{k}}^{2}\right\}$. These in turn depend upon the filters $\left\{H_{k}\right\}$. From (9) it follows that the required power $P_{k}$ in the $k$ th band is a linear (hence concave) function ${ }^{2}$ of $\sigma_{q_{k}}^{2}$. The total transmitted power $P$ is therefore a concave function of the noise variance vector

$$
\left[\begin{array}{llll}
\sigma_{q_{0}}^{2} & \sigma_{q_{1}}^{2} & \cdots & \sigma_{q_{M-1}}^{2}
\end{array}\right]^{T}
$$

From Fig. 2(c), we see that this is the vector of subband variances of the orthonormal filter bank $\left\{H_{k}\left(e^{j \omega}\right)\right\}$ in response to the power spectrum $S_{e e}\left(e^{j \omega}\right) /\left|C\left(e^{j \omega}\right)\right|^{2}$. Recalling the discussion on PCFBs from Section IV we now see that the orthonormal filter bank $\left\{H_{k}\left(e^{j \omega}\right)\right\}$ which minimizes total power for fixed error probabilities and bit rates is indeed a PCFB for the effective noise power spectrum

$$
S_{q q}\left(e^{j \omega}\right) \triangleq S_{e e}\left(e^{j \omega}\right) /\left|C\left(e^{j \omega}\right)\right|^{2}
$$

Having identified this PCFB, the variances $\sigma_{q_{k}}^{2}$ are readily computed, from which the powers $P_{\boldsymbol{k}}$ for fixed bit rate $b_{k}$ and error probability $\mathcal{P}_{e}(k)$ can be found [using (9)]. The minimized power $P$ can then be calculated.

\footnotetext{
${ }^{2} \mathrm{~A}$ linear function is also convex, so there is a permutation of the optimal PCFB which maximizes rather than minimizes power. Evidently it should be avoided!
} 


\section{B. Maximizing Total Bit Rate}

Returning to the error probability expression (8) let us now invert it to obtain a formula for the bit rate $b_{k}$. This is tricky because of the way $b_{k}$ occurs in two places. The factor $\left(1-2^{-b_{k}}\right)$ however is a weak function of $b_{k}$ in the sense that it varies from 0.5 to 1 as $b_{k}$ changes from one to infinity. Suppose we replace $\left(1-2^{-b_{k}}\right)$ with unity. Then, (8) yields

$$
b_{k}=0.5 \log _{2}\left(1+\frac{3}{\left[\mathcal{Q}^{-1}\left(\mathcal{P}_{e}(k) / 2\right)\right]^{2}} \frac{P_{k}}{\sigma_{q_{k}}^{2}}\right)
$$

so the total $b=\sum_{k} b_{k}$ is approximately

$$
b=0.5 \sum_{k=0}^{M-1} \log _{2}\left(1+\frac{3}{\left[\mathcal{Q}^{-1}\left(\mathcal{P}_{e}(k) / 2\right)\right]^{2}} \frac{P_{k}}{\sigma_{q_{k}}^{2}}\right) \text {. }
$$

This is the achievable $b$ without channel coding, for a given set of error probabilities $\left\{\mathcal{P}_{e}(k)\right\}$ and signal to noise ratios $\left\{P_{k} / \sigma_{q_{k}}^{2}\right\}$. Since $\log _{2}(1+a / x)$ is convex in $x$ (for $a, x>0$ ), the total bit rate is convex in the variance vector (14). Thus, the orthonormal filter bank $\left\{H_{k}\left(e^{j \omega}\right)\right\}$ which maximizes the bit rate for fixed error probabilities and powers is again a PCFB for the effective noise psd $S_{e e}\left(e^{j \omega}\right) /\left|C\left(e^{j \omega}\right)\right|^{2}$ as before. This is intuitively appealing since the maximization of bit rate and minimization of total power are consistent goals.

Without the approximation $1-2^{-b_{k}} \approx 1$ the closed form expression (16) is not possible, but the convexity of $b$ can still be proved in a more elaborate way as shown in Appendix B.

\section{Optimal Power Allocation}

The preceding result is true regardless of how the total power $P=\sum_{k} P_{k}$ is allocated among the bands. In particular we can perform optimum power allocation. We have

$$
b=0.5 \sum_{k=0}^{M-1} \log _{2}\left(1+\frac{P_{k}}{N_{k}}\right)
$$

where $N_{k}=\sigma_{q_{k}}^{2}\left[\mathcal{Q}^{-1}\left(\mathcal{P}_{e}(k) / 2\right)\right]^{2} / 3$. The optimization of $\left\{P_{k}\right\}$ for fixed total power $P=\sum_{k} P_{k}$ is a standard problem in information theory [11]. The solution is given by

$$
P_{k}= \begin{cases}\lambda-N_{k}, & \text { if this is nonnegative, } \\ 0, & \text { otherwise }\end{cases}
$$

where $\lambda$ is chosen to meet the power constraint. This is the familiar water pouring rule [11] demonstrated in Fig. 7. This power allocation is optimal regardless of the exact choice of the filter bank $\left\{H_{k}(z)\right\}$. In particular, if $\left\{H_{k}(z)\right\}$ is chosen as the optimal PCFB and then power is allocated as above, it provides the maximum possible DMT bit rate $b$ for fixed total power and fixed set of error probabilities. Note that the power allocation automatically determines bit allocation because of the formula (15).

\section{Capacity}

We observe some similarities and differences between the actual bit rate (16) and the theoretical capacity of the DMT system.

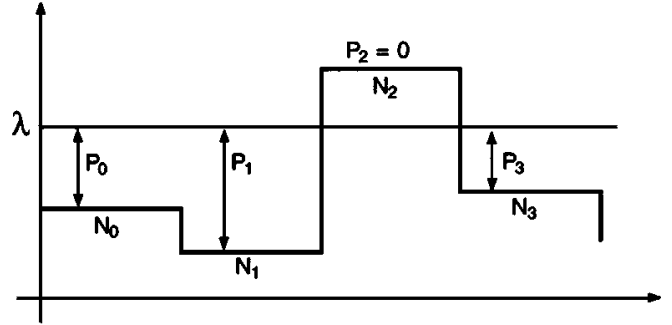

Fig. 7. Optimal power allocation by water pouring.

The biorthogonal DMT system with ideal channel equalizer can be represented by

$$
y_{k}(n)=x_{k}(n)+q_{k}(n)
$$

where $x_{k}(n)$ are the modulation symbols and $q_{k}(n)$ the noise components shown in Fig. 2(c). In general it is not true that the effective noise components $q_{k}(n)$ are Gaussian, white, and uncorrelated. However, if the number of bands $M$ is large, and the filters $H_{k}(z)$ are good approximations to ideal filters, then this is nearly the case. In this case, the channel (19) is identical to the parallel Gaussian channel and has information theoretic capacity [11]

$$
\mathcal{C}=0.5 \sum_{k=0}^{M-1} \log _{2}\left(1+\frac{P_{k}}{\sigma_{q_{k}}^{2}}\right) .
$$

Since the noise variances $\sigma_{q_{k}}^{2}$ depend on the filters $\left\{F_{k}, H_{k}\right\}$, the above capacity $\mathcal{C}$ also depends on them. For the case where $\left\{H_{k}\right\}$ is an orthonormal filter bank this capacity is maximized if $\left\{H_{k}\right\}$ is chosen as a PCFB for the effective noise psd $S_{e e}\left(e^{j \omega}\right) /\left|C\left(e^{j \omega}\right)\right|^{2}$. The reason again is that (20) is convex in the variance vector (14). Moreover, as in [11], we can optimally allocate the powers $P_{k}$ under a power constraint $P=\sum_{k} P_{k}$.

Equation (16) is the bit rate achieved for fixed probabilities of error $\left\{\mathcal{P}_{e}(k)\right\}$, and without channel-coding in subbands. Equation (20) is the information capacity, that is, the theoretical upper bound on achievable bit rate with arbitrarily small error. We see that both (16) and (20) depend on the choice of filter bank, and are maximized by the PCFB. Suppose the error probabilities are $\mathcal{P}_{e}(k)=10^{-7}$ for all $k$. A calculation of the factor $3 /\left[\mathcal{Q}^{-1}\left(\mathcal{P}_{e}(k) / 2\right)\right]^{2}$ shows that if the two quantities $b$ and $\mathcal{C}$ have to be equal then the total power in (16) should be $9.74 \mathbf{~ d B}$ more than the power used in (20). Channel coding is included in many DMT systems in order to reduce this gap. ${ }^{3}$

The preceding discussion on capacity should be interpreted carefully. Indeed, the capacity of a channel is a property of the channel itself, and cannot depend on the filter bank. It depends on the power, the channel transfer function, and the noise. However, in the preceding interpretation we imagine that the $M$-band transmitter filter bank and receiver filter bank are part of the channel. The number of bands $M$ and the powers $\left\{P_{k}\right\}$ are fixed, and the filter bank is assumed orthonormal. Under this condition, (20) represents the capacity of the channel, and it depends only on the noise variance distribution $\left\{\sigma_{q_{k}}^{2}\right\}$ which can be controlled by the receiver filters (the transmitter filters are

\footnotetext{
${ }^{3}$ This gap is very similar to the gap between PCM rate and channel capacity for AWGN channels [19, Ch. 15].
} 


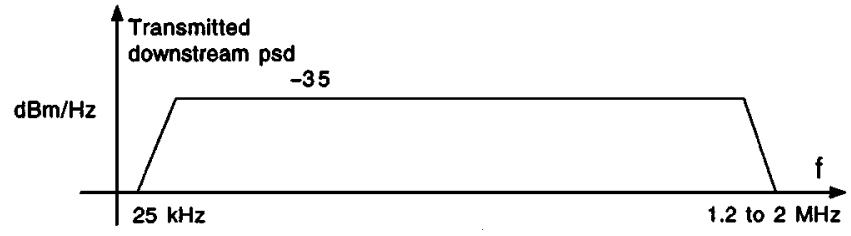

(a)

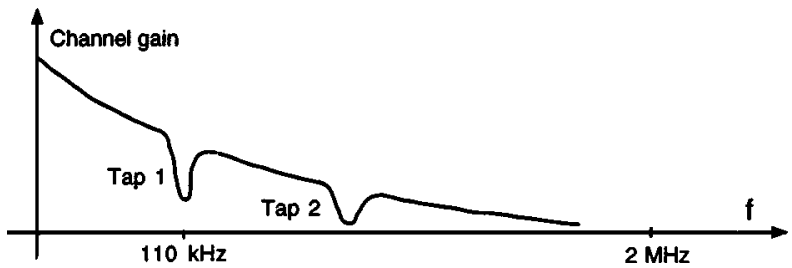

(c)

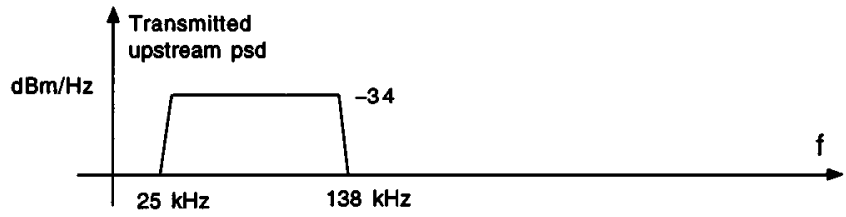

(b)

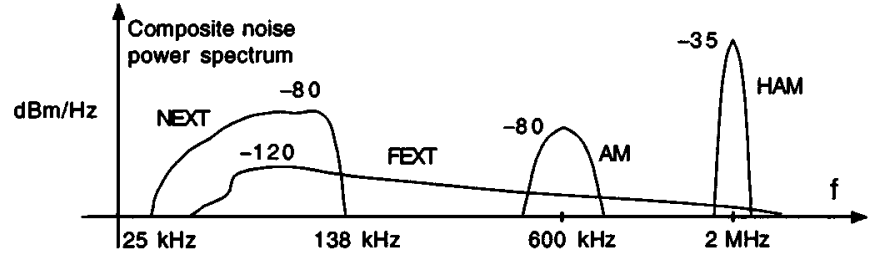

(d)

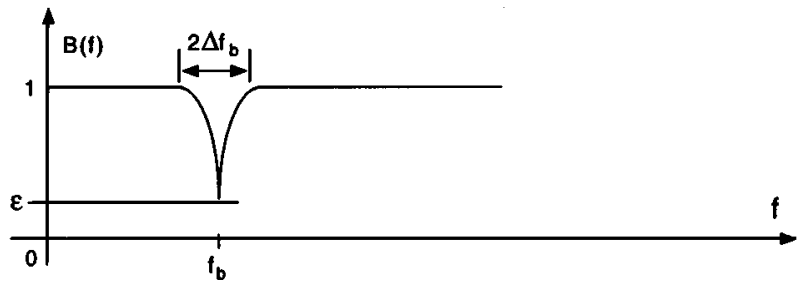

(e)

Fig. 8. Qualitative frequency-domain plots for ADSL service on the copper twisted-pair channel. (a) and (b) Transmitted psd masks; (c) Channel gain $\left|C_{c}(f)\right|^{2}$. (d) Various noise spectra. (e) Model for the bridged tap.

conjugates of the receiver filters by orthonormality and perfect reconstruction requirement). This is a useful interpretation because of the close analog between (20) and the bit rate (16). This capacity is maximized by choosing the filter bank to be a PCFB and by performing power allocation as described earlier. Notice finally that the bit rate equation (16) with nonzero error probability is both practical and perfectly meaningful, and is in no way affected by the preceding interpretation based on capacity which in this context is only of aesthetic value.

\section{TWISTED PAIR CHANNEL}

The data rate achievable on twisted-pair copper wires is limited by the channel noise and the gain of the line $\left|C_{c}(f)\right|^{2}$, which decreases with frequency and wire length. The signal to noise ratio deteriorates rapidly with frequency as well as wire length. Nevertheless, with typical noise sources of the kind encountered in a DSL environment and with typical transmitted power levels, a wire of length $18 \mathrm{kft}$ could achieve a rate well above $1 \mathrm{Mbits} / \mathrm{s}$. Shorter wires (e.g., $1 \mathrm{kft}$ ) can achieve much more (40-60 Mbits/s) [31], [43]. This is done by allocating power and bits into a much wider bandwidth than the traditional voice band.

The purpose of this section is to demonstrate the improvement obtainable with optimal filter banks instead of a DFT based DMT system. A simplified model of the twisted pair environment will be used. The model, while not accurate, helps to demonstrate the ideas well. Only a real simulation with published data on the channel can reveal the improvements more accurately, but we shall not venture into that here.

The types of noise that are really important in a DSL environment are near end cross talk or next and far end cross talk or fext. These arise because several twisted pairs are typically placed in a single cable and therefore suffer from electromagnetic interference from each other. A great deal of study has been done on this, both theoretical and measurement-based [31], [43]. Assuming that all the pairs in the cable are excited with the same input psd, the power spectra of the next and fext noise sources can be estimated using standard procedures. Even though the "next noise" is an interference, it has the characteristic of Gaussian noise as shown in [18].

Fig. 8 demonstrates the relevant quantities with plots that reasonably resemble what one might expect in practice. Parts (a) and (b) show the transmitted downstream (telephone office to customer) and upstream (customer to telephone office) power distributions for ADSL service. These signals often occupy nonoverlapping bands but sometimes they are in the same band, in which case echo cancelers are required [31]. The downstream bandwidth is larger because of higher rate (several megabits per second); upstream offers only a few hundred kilobits per second. Fig. 8(c) shows a typical plot of the channel gain. The dips are due to bridged taps typically attached to telephone lines in the US for service flexibility. Fig. 8(d) shows the typical power spectra of the next and fext noises. The figure also shows the typical interference on the phone line caused by AM radio waves $(560 \mathrm{kHz}-1.6 \mathrm{MHz})$ and from amateur radio (1.81-29.7 MHz, which is outside the standard ADSL band as deployed today). These interferences depend of course on the location of the line, time of the day and many other varying factors.

In any case, notice that the overall noise spectrum is far from flat. The ratio of the noise spectrum to the channel gain given by $S_{e e, c}(f) /\left|C_{c}(f)\right|^{2}$ is not monotone. Because of the many bumps and dips in this ratio, the PCFB is significantly different 
from the contiguous brickwall stacking, and can therefore reduce transmitted power significantly, similar to the example of Section III. This is demonstrated next.

\section{A. Details}

We assume that the channel gain $\left|C_{c}(f)\right|^{2}$ as a function of the continuous-time frequency $f$ has the form [16]

$$
\left|C_{c}(f)\right|^{2}=\beta e^{-\alpha \sqrt{f} \ell / \ell_{0}}
$$

where $\ell$ is the length of the twisted pair line in kilofeet and $f$ is in kilohertz. The constants appearing in the equation are $\alpha=1.158, \beta=10^{-1.2}$, and $\ell_{0}=18$. Notice that this value of $\beta$ yields an attenuation of $12 \mathrm{~dB}$ at zero frequency. The preceding expression for $\left|C_{c}(f)\right|^{2}$ is sometimes referred to as the RC-approximation, and is valid for short lengths [16]. We approximate each bridged tap with a multiplicative term $B(f)$ having the form shown in shown in Fig. 8(e). The expression used in the simulation is

$$
B(f)= \begin{cases}1, & \left|f-f_{b}\right|>\Delta f_{b} \\ 1-\frac{1-\epsilon}{\Delta f_{b}^{2}}\left(\left|f-f_{b}\right|-\Delta f_{b}\right)^{2}, & \text { otherwise. }\end{cases}
$$

This expression is used for $f \geq 0$, and it defines $B(f)$ for all $f$ because $B(-f)=B(f)$. The center frequency $f_{b}$ is determined by the length of the bridged tap. The noise psd $S_{e e, c}(f)$ as a function of continuous-time frequency $f$ has the form

$$
S_{e e, c}(f)=S_{\text {next }}(f)+S_{\text {fext }}(f)+S_{\mathrm{am}}(f) .
$$

For simplicity, the AM noise psd $S_{\text {am }}(f)$ for a given station is assumed to be a constant with total bandwidth of $10 \mathrm{kHz}$ around the station frequency $f_{\text {am }}$. Its strength $10 \log _{10} S_{\text {am }}(f)$ can be specified in $\mathrm{dB} \cdot \mathrm{m} / \mathrm{Hz}$ (typically between -80 and -120 $\mathrm{dB} \cdot \mathrm{m} / \mathrm{Hz}$ on phone lines [31]). We consider the ADSL downstream channel for which the next and fext sources are, respectively, the upstream and downstream signals in the other twisted pairs in the cable. We assume the upstream and downstream signal power spectra $S_{\mathrm{up}}(f)$ and $S_{\mathrm{dn}}(f)$ to be as in the ADSL issue 2 mask described in [31]. More specifically, these are taken to be the plots on pages 103 and 105 of [31] multiplied by the baseband pulse shaping function

$$
\left(\frac{\sin \left(\pi f / f_{0}\right)}{\pi f / f_{0}}\right)^{2}
$$

where $f_{0}=2.208 \mathrm{MHz}$ for downstream and $270 \mathrm{kHz}$ for upstream [31]. The psd of next and fext noise sources are taken to be

$$
\begin{aligned}
S_{\text {next }}(f) & =c_{n}\left(N_{n} / 49\right)^{0.6} f^{1.5} S_{\mathrm{up}}(f) \\
S_{\text {fext }}(f) & =c_{f}\left(N_{f} / 49\right)^{0.6} f^{2} \ell\left|C_{c}(f)\right|^{2} B(f) S_{\mathrm{dn}}(f)
\end{aligned}
$$

where $c_{n}=10^{-13}$ and $c_{f}=9 \times 10^{-17}$. Here $\ell$ is the wire length in kilofeet and $f$ is in hertz. The integers $N_{n}$ and $N_{f}$ are the number of next and fext disturbers ( $\leq 49$ in a 50-pair cable). For our example, we assume the following.
1) Number of subchannels $M=8$, sampling rate $=$ 3.2 $\mathrm{MHz}$, and probabilities of error $\mathcal{P}_{e}=10^{-9}$ in all subchannels. PAM constellations are used in each subchannel.

2) Twisted pair channel length $\ell=3 \mathrm{kft}$, and number of disturbers $=49$ (for both next and fext).

3) One bridged tap with $\epsilon=1 / 60, \Delta f_{b}=150 \mathrm{~Hz}$ and $f_{b}=123 \mathrm{kHz}$.

4) Two AM stations with $\mathrm{BW} 10 \mathrm{kHz}$ each, having carrier frequencies 600 and $850 \mathrm{kHz}$ with power spectra -95 $\mathrm{dB} \cdot \mathrm{m} / \mathrm{Hz}$ and $-90 \mathrm{~dB} \cdot \mathrm{m} / \mathrm{Hz}$, respectively.

Then, for a downstream ADSL bit rate of $3.2 \mathrm{Mbits} / \mathrm{s}$, the transmitted power is required to have the following values:

$\begin{array}{ll}\text { Traditional DFT-multitone: } & 4.68 \mathrm{~mW} \\ \text { DCT-multitone: } & 4.08 \mathrm{~mW} \\ \text { KLT-multitone: } & 2.76 \mathrm{~mW} \\ \text { Ideal FB [contiguous stacking, Fig. 3(b)]: } & 1.28 \mathrm{~mW} \\ \text { Ideal PCFB (unconstrained class } \mathcal{C}_{u} \text { ): } & 0.94 \mathrm{~mW} .\end{array}$

The PCFB is, therefore, significantly better than the other filter banks. Compared to the traditional DFT, the savings in power is about a factor of five. Fig. 9 shows the responses of two of the eight filters in the PCFB (normalized to unity). Notice that the filters have multiple passbands. The plot for $H_{4}\left(e^{j \omega}\right)$ shows that its practical implementation could be expensive because of the very narrow passbands. In fact, by a slight variation of the PCFB design algorithm, it is possible to eliminate bands that are narrower than a certain threshold. Such near-PCFB solutions will still have performance close to ideal. In any case, it is our opinion that the primary role of the PCFB is to provide bounds on performance for fixed $M$. If the performance gap between a practical system and the PCFB solution is small in a particular application, this gives the assurance that we are not very far from optimality.

If we plot the required transmitted power as a function of the number of bands $M$ (with all other parameters as in the previous example) the result is as shown in Fig. 10. The plot shows the results for $M=1,2,4,8,16,32,64$, and 128 . The PCFB requires smaller power than all other methods (consistent with its theoretical optimality). However, the difference between various filter banks becomes negligible as $M$ increases. This is analogous to a well-known observation in subband coding [14]; namely the coding gain is relatively insensitive to the choice of filter bank as $M \rightarrow \infty$. DMT systems based on fixed filter banks such as the DFT or cosine modulated filter banks are attractive because of their simplicity; they are non adaptive and can be implemented efficiently [10], [27].

\section{SCALAR PREFILTERING Before ChanNel}

Consider again, Fig. 1 where $\left\{H_{k}\right\}$ is orthonormal with $F_{k}\left(e^{j \omega}\right)=H_{k}^{*}\left(e^{j \omega}\right)$. Assume as before that $C(z)$ has been equalized by inserting $1 / C(z)$. Suppose this configuration is further modified by insertion of a prefilter and postfilter around the channel [Fig. 11(a)]. Thus the effective transmitting filters are $F_{k}^{\prime}(z)=F_{k}(z) D(z)$ and receiving filters are $H_{k}^{\prime}(z)=H_{k}(z) / D(z)$. This defines a biorthogonal filter bank $\left\{F_{k}^{\prime}(z), H_{k}^{\prime}(z)\right\}$. This system can achieve better performance 


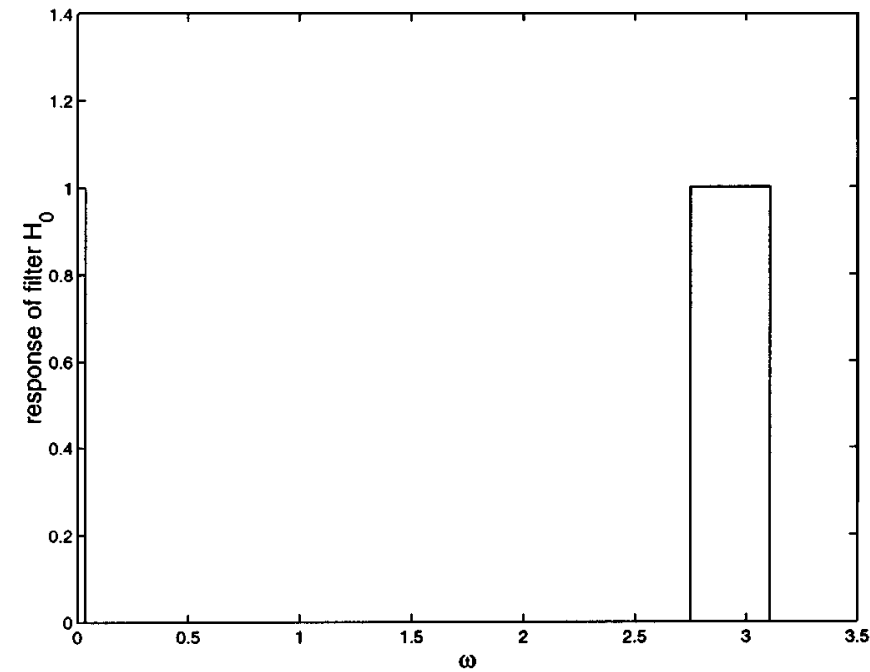

(a)

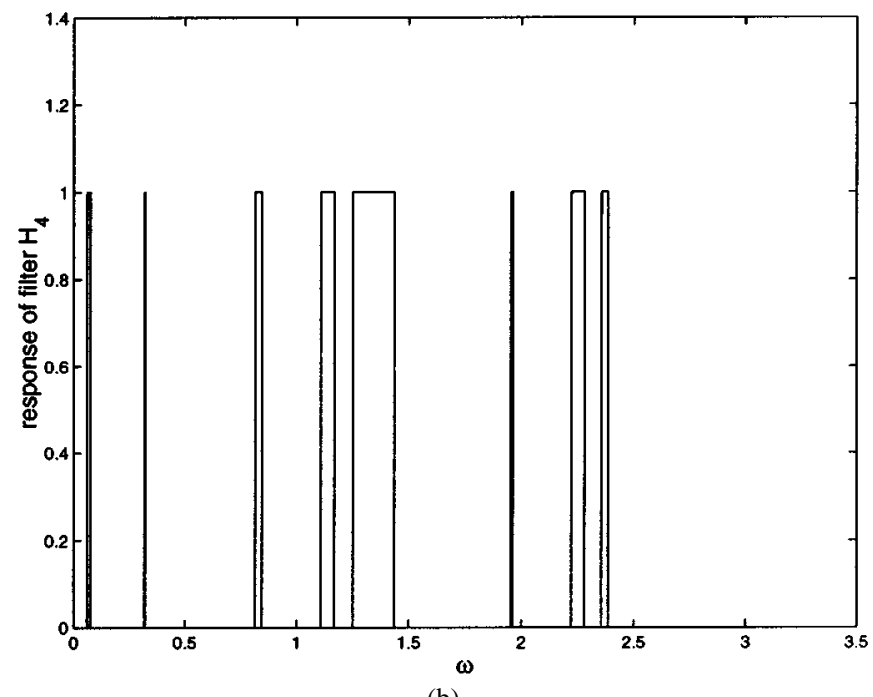

(b)

Fig. 9. Two of the $M$ filters in the PCFB which minimizes transmitted power in the example.

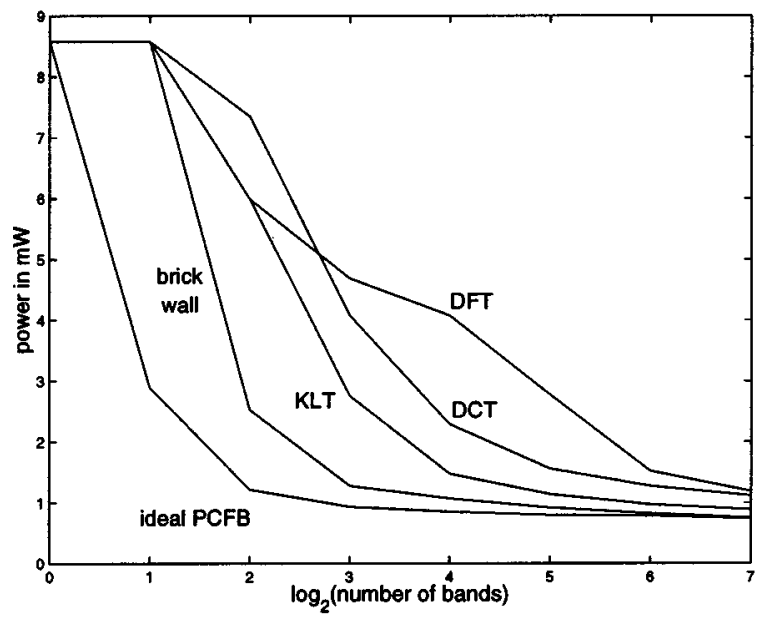

Fig. 10. A comparison of the PCFB with other filter banks as a function of number of bands $M$.

than the orthonormal system $\left\{F_{k}(z), H_{k}(z)\right\}$. For example, we can shape $D(z)$ and $\left\{F_{k}(z)\right\}$ such that the transmitted power is minimized for fixed bit rates and probabilities of error.
The interpolated signal $s_{k}(n)$ [Fig. 11(b)] has a variance which in general depends on $n$. In fact, if we assume that $x_{k}(n)$ is a WSS process, the signal $s_{k}(n)$ is cyclo WSS, and its variance is a periodic function of $n$ with period $M$. The power in the $k$ th symbol is this variance averaged over a period. To find this, redraw Fig. 11(b) as in Fig. 11(c) where $R_{n k}(z)$ are the polyphase components of $F_{k}(z) D(z)$. We shall assume that the symbols $x_{k}(n)$ are white with zero mean and variance $P_{k}$. This is consistent with the view that $x_{k}(n)$ is generated by parsing a binary i.i.d. sequence [8]. Thus the variance at the output of $R_{n k}(z)$ is given by $\int P_{k}\left|R_{n k}\left(e^{j \omega}\right)\right|^{2} d \omega / 2 \pi$. The average variance of $s_{k}(n)$ is then

$$
\begin{aligned}
& \frac{P_{k}}{M} \sum_{n=0}^{M-1} \int_{0}^{2 \pi}\left|R_{n k}\left(e^{j \omega}\right)\right|^{2} d \omega / 2 \pi \\
& =\frac{P_{k}}{M} \int_{0}^{2 \pi}\left|F_{k}\left(e^{j \omega}\right) D\left(e^{j \omega}\right)\right|^{2} d \omega / 2 \pi
\end{aligned}
$$

Assuming further that $x_{k}(n)$ are uncorrelated for different $k$, the total power input to the channel is the sum of these average variances

$$
P=\frac{1}{M} \sum_{k=0}^{M-1} P_{k} \int_{0}^{2 \pi}\left|F_{k}\left(e^{j \omega}\right) D\left(e^{j \omega}\right)\right|^{2} d \omega / 2 \pi .
$$

The quantity $P_{k}$ is also the physical signal-power at the input of the detector. The noise variance $\sigma_{q_{k}}^{2}$ at the detector input can be computed by referring to Fig. 2(c) and inserting the additional factor $1 / D(z)$ in the noise transfer functions. Thus

$$
\sigma_{q_{k}}^{2}=\int_{0}^{2 \pi} \frac{S_{e e}\left(e^{j \omega}\right)\left|H_{k}\left(e^{j \omega}\right)\right|^{2}}{\left|C\left(e^{j \omega}\right) D\left(e^{j \omega}\right)\right|^{2}} d \omega / 2 \pi
$$

Since $P_{k}=g\left[b_{k}, \mathcal{P}_{e}(k)\right] \sigma_{q_{k}}^{2}$ for some $g[.,$.$] , the total power is$

$$
\begin{aligned}
P=\frac{1}{M} \sum_{k=0}^{M-1} g\left[b_{k}, \mathcal{P}_{e}(k)\right] \int_{0}^{2 \pi} & \frac{S_{e e}\left(e^{j \omega}\right)\left|F_{k}\left(e^{j \omega}\right)\right|^{2}}{\left|C\left(e^{j \omega}\right) D\left(e^{j \omega}\right)\right|^{2}} \frac{d \omega}{2 \pi} \\
& \times \int_{0}^{2 \pi}\left|F_{k}\left(e^{j \omega}\right) D\left(e^{j \omega}\right)\right|^{2} \frac{d \omega}{2 \pi}
\end{aligned}
$$

where we have substituted the preceding expression for $\sigma_{q_{k}}^{2}$ and used the fact that $H_{k}\left(e^{j \omega}\right)=F_{k}^{*}\left(e^{j \omega}\right)$ for any orthonormal filter bank. For a given channel, $C\left(e^{j \omega}\right)$ and $S_{e e}\left(e^{j \omega}\right)$ are fixed. Assume the set of error probabilities $\left\{\mathcal{P}_{e}(k)\right\}$ and bit rates $\left\{b_{k}\right\}$ are also fixed. The total power input to the channel then depends on the orthonormal filter bank $\left\{F_{k}\left(e^{j \omega}\right)\right\}$ and the prefilter $D\left(e^{j \omega}\right)$. The next result shows how this power can be minimized. It is an extension to the DMT system, of a familiar result in the subband coding theory [12].

Theorem 1: Optimum Prefiltered Orthonormal FB for $D M T$ : Assume that the modulation symbols $x_{k}(n)$ are white, and uncorrelated for different $k$. For fixed probabilities of error $\mathcal{P}_{e}(k)$ and bit rates $b_{k}$, the combination of orthonormal filter bank $\left\{H_{k}\right\}$ and prefilter $D(z)$ which minimizes the total required power $P$ is obtained as follows: 1) Choose $D(z)$ with magnitude response (22); 2) Make $\left\{H_{k}\right\}=$ PCFB for

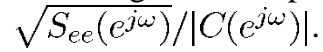




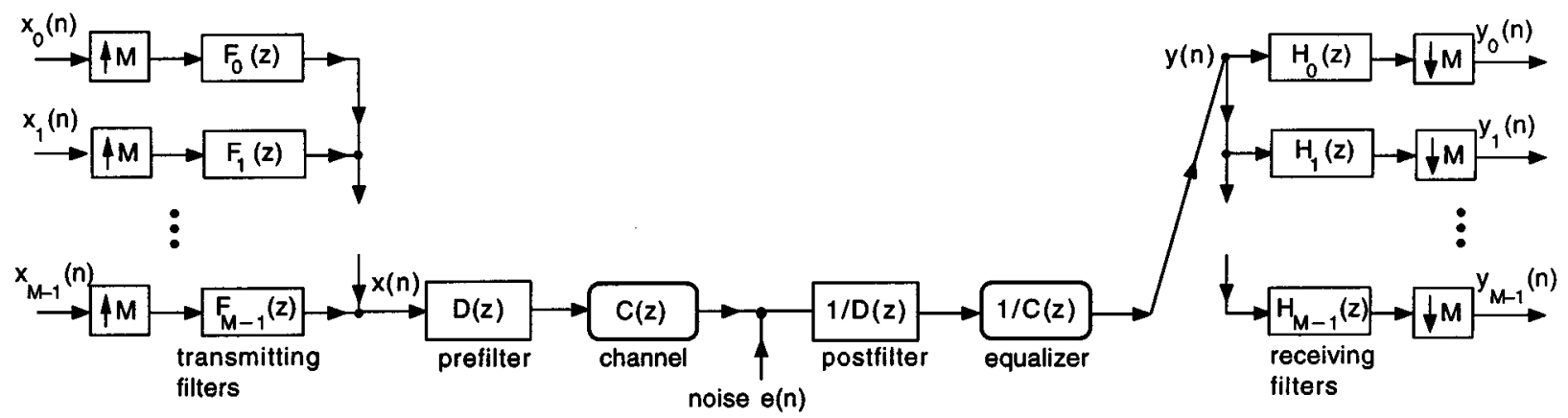

(a)

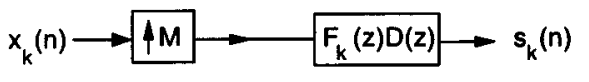

(b)

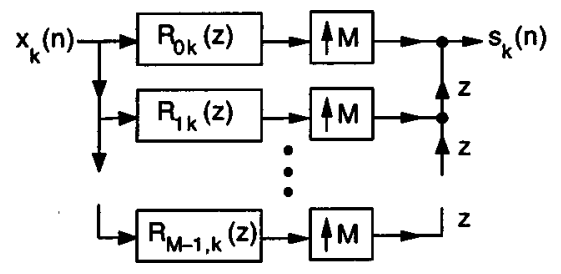

(c)

Fig. 11. (a) Pre and post filters in the DMT system. (b) The $k$ th subchannel symbol and its interpolation. (c) Equivalent polyphase diagram.

Proof: From the Cauchy-Schwartz inequality we have

$$
\int \frac{S_{e e}\left|F_{k}\right|^{2}}{|C D|^{2}} \frac{d \omega}{2 \pi} \int\left|F_{k} D\right|^{2} \frac{d \omega}{2 \pi} \geq\left(\int \frac{\sqrt{S_{e e}}\left|F_{k}\right|^{2}}{|C|} \frac{d \omega}{2 \pi}\right)^{2}
$$

where the argument $\left(e^{j \omega}\right)$ has been eliminated for simplicity. Equality holds when the two integrands on the left are equal, that is,

$$
\left|D\left(e^{j \omega}\right)\right|=\left(S_{e e}\left(e^{j \omega}\right) /\left|C\left(e^{j \omega}\right)\right|^{2}\right)^{1 / 4}
$$

This is the optimum $\left|D\left(e^{j \omega}\right)\right|$ no matter what the orthonormal filter bank $\left\{H_{k}\right\}$ is. With the prefilter chosen as above, the total transmitted power is $P=\sum_{k=0}^{M-1} g\left[b_{k}, \mathcal{P}_{e}(k)\right] \eta_{k}^{2} / M$ where

$$
\eta_{k}^{2}=\left(\int_{0}^{2 \pi} \frac{\sqrt{S_{e e}\left(e^{j \omega}\right)}\left|H_{k}\left(e^{j \omega}\right)\right|^{2}}{\left|C\left(e^{j \omega}\right)\right|} d \omega / 2 \pi\right)^{2}
$$

Thus, $P$ is a concave function of $\left[\begin{array}{llll}\eta_{0}^{2} & \eta_{1}^{2} & \cdots & \eta_{M-1}^{2}\end{array}\right]^{T}$ which can be regarded as a subband variance vector from an orthonormal analysis bank with input power spectrum $\sqrt{S_{e e}\left(e^{j \omega}\right)} /\left|C\left(e^{j \omega}\right)\right|$. Applying the result of Section IV we conclude that the orthonormal filter bank $\left\{H_{k}\right\}$ minimizing the total power is a PCFB for the power spectrum $\sqrt{S_{e e}\left(e^{j \omega}\right)} /\left|C\left(e^{j \omega}\right)\right|$.

$\nabla \nabla \nabla$

Note that the solution (22) also arises in optimal prefiltering prior to scalar quantization, and is said to be the half whitening filter [14], [36] for the spectrum $\left|C\left(e^{j \omega}\right)\right|^{2} / S_{e e}\left(e^{j \omega}\right)$.

\section{CONCLUDING REMARKS}

The DMT idea is similar in principle to subband coding where a signal $x(n)$ to be quantized is first decomposed into subbands. Depending on the power spectrum $S_{x x}\left(e^{j \omega}\right)$ of the input, there is a certain distribution of signal energy across the subbands. This distribution is exploited in the coding process by optimal bit allocation: we allocate more bits to the subband having higher energy. Thus, in the subband coder, the frequency dependence of the input signal $x(n)$ is exploited. In the DMT system, the frequency dependence of the channel $C\left(e^{j \omega}\right)$ and the noise $S_{e e}\left(e^{j \omega}\right)$ are exploited. The similarity of the two problems is exemplified by the fact that the PCFB serves as an optimal theoretical benchmark in both cases. The complete duality between the optimization of subband coders and DMT systems can also be seen in a more basic way as explained in [21]. The use of nonuniform filter banks and PCFBs for DMT communication has not been addressed in this paper. Such an extension finds application in the so-called DWMT (discrete wavelet multitone) modulation. We conclude with one further remark. The implicit assumption throughout has been that the channel and the noise power spectrum are entirely known so that the optimal filter bank can be identified. If there is an error in the estimation of these channel parameters, then naturally the performance would be suboptimal. An interesting research problem in this context would be to analyze the extent to which the results will stray from optimality.

\section{APPENDIX A \\ PARsing Stage in DMT COMMUNiCATION}

Fig. 12(a) shows the first stage of multitone modulation [8], [10] called the parsing stage. Here $s(n)$ represents binary data to be transmitted over a channel. This data is divided into nonoverlapping $b$-bit blocks. The $b$ bits in each block are partitioned into $M$ groups, the $k$ th group being a collection of $b_{k}$ bits (demonstrated in the figure for $M=3$ ). Thus, the total number of bits $b$ per block can be expressed as $b=\sum_{k=0}^{M-1} b_{k}$.

The $b_{k}$ b in the $k$ th group constitute the $k$ th symbol $x_{k}$ which can therefore be regarded as a $b_{k}$-b number. For the $n$th block, this symbol is denoted as $x_{k}(n)$. This is the modulation symbol for the $k$ th band. The vector $\left[x_{0}(n) x_{1}(n) \cdots x_{M-1}(n)\right]^{T}$ is sometimes referred to as the DMT symbol. For the case of pulse amplitude modulation (PAM), the sample $x_{k}(n)$ is a quantized real number as demonstrated in Fig. 12(c) for $b_{k}=3$. For the 


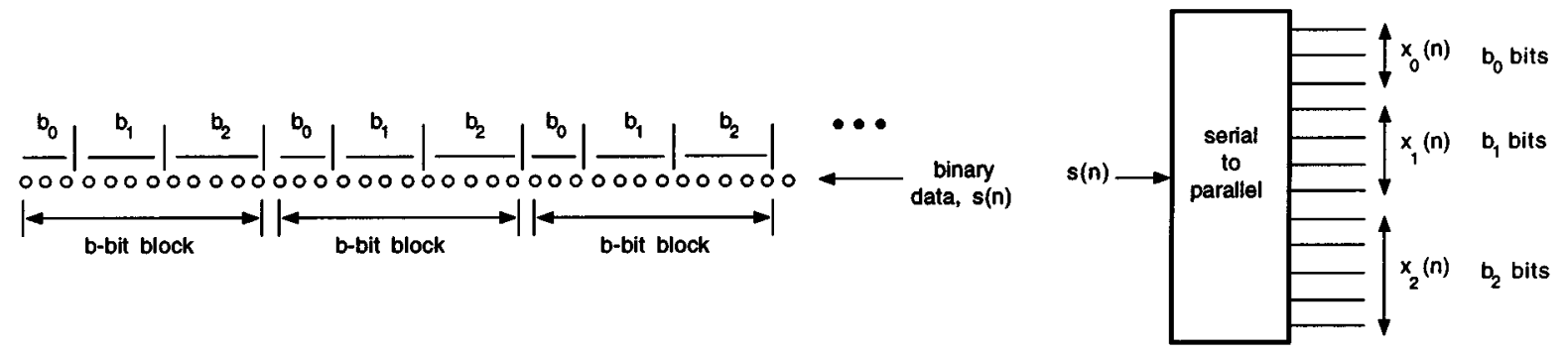

(a)

(b)

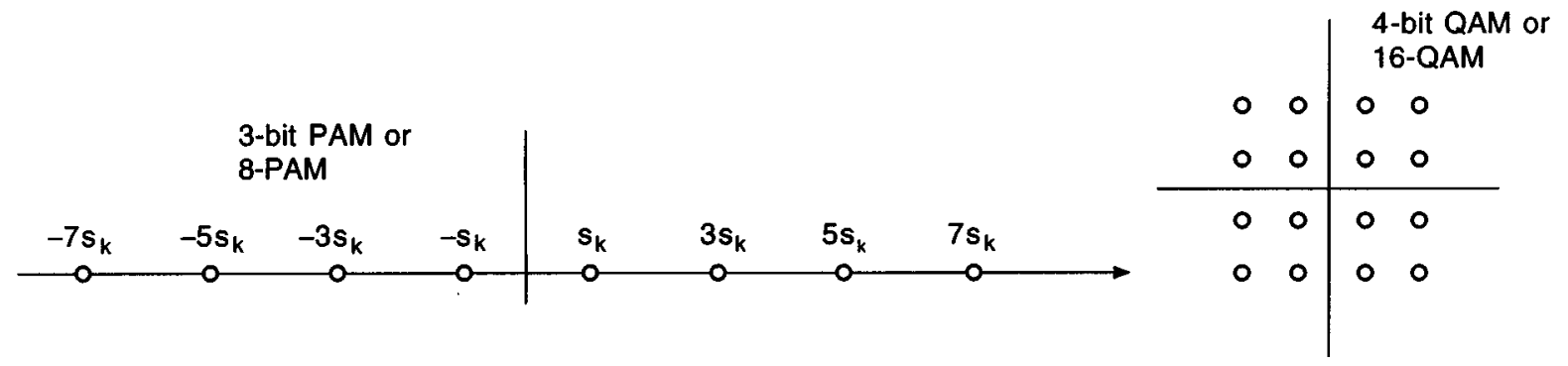

(c)

(d)

Fig. 12. (a) and (b) Explanation of the parsing stage in DMT. (c) The 8-PAM constellation (3 b). (d) the 16-QAM constellation (4 b).

case of quadrature amplitude modulation (QAM) $x_{k}(n)$ can be regarded as a complex number, taking one of $2^{b_{k}}$ possible values from a rectangular constellation as demonstrated in Fig. 12(d). More efficient constellations exist [26].

\section{APPENDIX B}

\section{Proof of CONVEXITY of BIT RATE}

The following proof was first presented in [6]. Consider (8) and delete all dependence on $k$ for simplicity. Without using the approximation $1-2^{-b} \approx 1$ we will show that $b$ is convex in $\sigma_{q}^{2}$. First notice that

$$
\frac{\sigma_{q}^{2}}{3 P}=g(b)=\left[\mathcal{Q}^{-1}\left(\frac{\mathcal{P}_{e}}{2\left(1-2^{-b}\right)}\right)\right]^{-2} \frac{1}{2^{2 b}-1}
$$

As $b$ increases from $b_{\min }=-\log _{2}\left(1-\mathcal{P}_{e}\right)$ to $\infty$, the quantity $g(b)$ decreases from $\infty$ to zero. We will show that $g(b)$ is convex for $b_{\min }<b<\infty$. Since the inverse of a decreasing convex function is convex (Appendix $\mathrm{C}$ ), this will prove that $b=g^{-1}\left(\sigma_{q}^{2} / 3 P\right)$ is convex in $\sigma_{q}^{2}$. For convenience define

$$
h(b)=\left[\mathcal{Q}^{-1}\left(\mathcal{P}_{e} /\left[2\left(1-2^{-b}\right)\right]\right)\right]^{2} .
$$

Then, $g(b)=1 /\left[h(b)\left(2^{2 b}-1\right)\right]$, and $-d g(b) / d b$ becomes

$$
\frac{h^{\prime}(b)}{h^{2}(b)\left(2^{2 b}-1\right)}+\frac{2 \log _{e} 2}{h(b)\left(2^{2 b}-1\right)}+\frac{2 \log _{e} 2}{h(b)\left(2^{2 b}-1\right)^{2}}
$$

where the primes denote derivatives with respect to $b$. We know $g(b)$ is convex if its second derivative is nonnegative. So it is sufficient to show that $-g^{\prime}(b)$ is decreasing. Both $1 / h(b)$ and $1 /\left(2^{2 b}-1\right)$ are positive and decreasing in $b_{\min }<b<\infty$, and so $h^{\prime}(b) \geq 0$ as well. It is therefore sufficient to show that $h^{\prime}(b) / h(b)$ decreases. Since $\mathcal{Q}(b)=\int_{b}^{\infty} e^{-u^{2} / 2} d u / \sqrt{2 \pi}$, it follows that $d \mathcal{Q}(b) / d b=-e^{-b^{2} / 2} / \sqrt{2 \pi}$. Similarly, the func- tion $b=Q^{-1}(v)$ has derivative $d b / d v=-\sqrt{2 \pi} e^{\left[\mathcal{Q}^{-1}(v)\right]^{2} / 2}$. Using these we verify that

$$
\frac{h^{\prime}(b)}{h(b)}=\frac{4 \sqrt{2 \pi}\left(\log _{e} 2\right) \mathcal{Q}(x) e^{x^{2} / 2}}{\mathcal{P}_{e}} \times\left(\frac{Q(x)-\left(\mathcal{P}_{e} / 2\right)}{x}\right)
$$

where $x \triangleq \mathcal{Q}^{-1}\left[\left(\mathcal{P}_{e} / 2\right) /\left(1-2^{-b}\right)\right]$. Now the range $b_{\min }<$ $b<\infty$ translates to $0<x<\mathcal{Q}^{-1}\left(\mathcal{P}_{e} / 2\right)$. In this range, $\left[\mathcal{Q}(x)-\left(\mathcal{P}_{e} / 2\right)\right] / x$ is decreasing. So, it is sufficient to show that $\mathcal{Q}(x) e^{x^{2} / 2}$ is decreasing in $x$, or its derivative is negative. This is equivalent to showing that $\sqrt{2 \pi} \mathcal{Q}(x)<e^{-x^{2} / 2} / x$. Now

$$
\sqrt{2 \pi} \mathcal{Q}(x)=\int_{x}^{\infty} e^{-y^{2} / 2} d y=-\int_{x}^{\infty} \frac{d\left(e^{-y^{2} / 2}\right)}{y} .
$$

Using integration by parts this indeed becomes

$$
\sqrt{2 \pi} \mathcal{Q}(x)=\frac{e^{-x^{2} / 2}}{x}-\int_{x}^{\infty} \frac{e^{-y^{2} / 2}}{y^{2}} d y<\frac{e^{-x^{2} / 2}}{x} .
$$

\section{APPENDIX C \\ DECREASING CONVEX FUNCTIONS}

To verify that the inverse of a decreasing convex function is convex, let $y=f(x)$ be an invertible convex function (in some range $x \in \mathcal{R})$. We have $f\left(\mu x_{0}+(1-\mu) x_{1}\right) \leq \mu f\left(x_{0}\right)+$ $(1-\mu) f\left(x_{1}\right)$ for $0 \leq \mu \leq 1$. Substituting $y_{0}=f\left(x_{0}\right)$ and $x_{0}=f^{-1}\left(y_{0}\right)$, and similarly for $y_{1}$, we get

$$
f\left(\mu f^{-1}\left(y_{0}\right)+(1-\mu) f^{-1}\left(y_{1}\right)\right) \leq \mu y_{0}+(1-\mu) y_{1} .
$$

If $f($.$) is a decreasing function, then this implies$

$$
\mu f^{-1}\left(y_{0}\right)+(1-\mu) f^{-1}\left(y_{1}\right) \geq f^{-1}\left(\mu y_{0}+(1-\mu) y_{1}\right)
$$

proving that $f^{-1}(y)$ is convex as well.

$\nabla \nabla \nabla$ 


\section{REFERENCES}

[1] A. N. Akansu, P. Duhamel, X. Lin, and M. de. Courville, "Orthogonal transmultiplexers in communications: A review," IEEE Trans. Signal Processing, vol. 46, pp. 979-995, Apr. 1998.

[2] S. Akkarakaran and P. P. Vaidyanathan, "On optimization of filter banks with denoising applications," in Proc. IEEE ISCAS'99, Orlando, FL, June 1999.

[3] _ - "The role of principal component filter banks in noise reduction," in Proc. SPIE, Denver, CO, July 1999.

[4] — - "Filter bank optimization with convex objectives, and the optimality of principal component forms," IEEE Trans. Signal Processing, vol. 49, pp. 100-114, Jan. 2001.

[5] _ _ "Results on principal component filter banks: Colored noise suppression and existence issues," IEEE Trans. Inform. Theory, to be published.

[6] - "Discrete multitone communication with principal component filter banks," in Proc. IEEE Int. Conf. Communications (ICC), Helsinki, Finland, June 2001

[7] N. Al-Dhahir and J. M. Cioffi, "Block transmission over dispersive channels: Transmit filter optimization and realization, and MMSE-DFE receiver performance," IEEE Trans. Inform. Theory, vol. 42, pp. 137-160, Jan. 1996.

[8] J. A. C. Bingham, "Multicarrier modulation for data transmission: An idea whose time has come," IEEE Commun. Mag., pp. 5-14, May 1990.

[9] G. Cherubini, E. Eleftheriou, S. Olcer, and J. M. Cioffi, "Filter bank modulation techniques for very high speed digital subscriber lines," IEEE Commun. Mag., pp. 98-104, May 2000.

[10] J. S. Chow, J. C. Tu, and J. M. Cioffi, "A discrete multitone transreceiver system for HDSL applications," IEEE J. Select. Areas Commun., vol. 9 , pp. 895-908, Aug. 1991.

[11] T. M. Cover and J. A. Thomas, Elements of Information Theory. New York: Wiley, 1991.

[12] I. Djokovic and P. P. Vaidyanathan, "On optimal analysis/synthesis filters for coding gain optimization," IEEE Trans. Signal Processing, vol. 44, pp. 1276-1279, May 1996.

[13] G. B. Giannakis, "Filter banks for blind channel identification and equalization," IEEE Signal Processing Lett., vol. 4, pp. 184-187, June 1997.

[14] N. S. Jayant and P. Noll, Digital Coding of Waveforms. Englewood Cliffs, NJ: Prentice-Hall, 1984

[15] I. Kalet, "The multitone channel," IEEE Trans. Commun., vol. 37, pp. 119-124, Feb. 1989.

[16] I. Kalet and S. Shlomo, "On the capacity of a twisted-wire pair: Gaussian model," IEEE Trans. Commun., vol. 38, pp. 379-383, Mar. 1990.

[17] I. Kalet, "Multitone modulation," in Subband and Wavelet Transforms, A. N. Akansu and M. J. Smith, Eds. Norwell, MA: Kluwer , 1996.

[18] J. K. Kerpez, "Near-end crosstalk is almost Gaussian," IEEE Trans. Commun., vol. 41, pp. 670-672, Jan. 1993.

[19] B. P. Lathi, Modern Digital and Analog Communication Systems. Oxford, U.K.: Oxford Univ. Press, 1998.

[20] X. Lin and A. N. Akansu, "A distortion analysis and optimal design of orthonormal basis for DMT receivers," in Proc. ICASSP'96, 1996, pp. $1475-1478$.

[21] Y. P. Lin, P. P. Vaidyanathan, S. Akkarakaran, and S.-M. Phoong, "On the duality of optimal DMT systems and biorthogonal subband coders," in Proc. ICASSP'00, Istanbul, Turkey, June 2000.

[22] Y.-P. Lin and S.M. Phoong, "Perfect discrete multitone modulation with optimal transceivers," IEEE Trans. Signal Processing, vol. 48, pp. 1702-1712, June 2000

[23] P. Moulin, "A new look at signal-adapted QMF bank design," in Proc. ICASSP'95, Detroit, MI, May 1995, pp. 1312-1315.

[24] P. Moulin, M. Anitescu, and K. Ramchandran, "Theory of rate-distortion optimal, constrained filter banks-Applications to IIR and FIR biorthogonal designs," IEEE Trans. Signal Processing, vol. 48, pp. 1120-1132, Apr. 2000

[25] D. Pal, G. N. Iyengar, and J. M. Cioffi, "A new method of channel shortening with applications to discrete multitone (DMT) systems," in Proc. IEEE Int. Conf. Communications, vol. 2, 1998, pp. 763-768.

[26] J. G. Proakis, Digital Communications. New York: McGraw Hill, 1995.

[27] A. D. Rizos, J. G. Proakis, and T. Q. Nguyen, "Comparison of DFT and cosine modulated filter banks in multicarrier modulation," in Proc. Globecom'94, Nov. 1994, pp. 687-691.

[28] A. Scaglione, G. B. Giannakis, and S. Barbarossa, "Redundant filter bank precoders and equalizers Part I: Unification and optimal designs," IEEE Trans. Signal Processing, vol. 47, pp. 1988-2006, July 1999.
[29] — - "Redundant filter bank precoders and equalizers-Part II: Synchronization and direct equalization," IEEE Trans. Signal Processing, vol. 47, pp. 2007-2022, July 1999.

[30] A. Scaglione, S. Barbarossa, and G. B. Giannakis, "Filterbank transceivers optimizing information rate in block transmissions over dispersive channels," IEEE Trans. Inform. Theory, vol. 45, pp. 1019-1032, Apr. 1999.

[31] T. Starr, J. M. Cioffi, and P. J. Silverman, Understanding Digital Subscriber Line Technology. Englewood Cliffs, NJ: Prentice-Hall, 1999.

[32] M. K. Tsatsanis and G. B. Giannakis, "Principal component filter banks for optimal multiresolution analysis," IEEE Trans. Signal Processing, vol. 43, pp. 1766-1777, Aug. 1995

[33] M. A. Tzannes, M. C. Tzannes, J. G. Proakis, and P. N. Heller, "DMT systems, DWMT systems, and digital filter banks," in Proc. ICC'94, 1994, pp. 311-315.

[34] M. Unser, "On the optimality of ideal filters for pyramid and wavelet signal approximation," IEEE Trans. Signal Processing, vol. 41, pp. 3591-3596, Dec. 1993.

[35] — - "An extension of the KLT for wavelets and perfect reconstruction filter banks," in Proc. Conf. Wavelet Applications in Signal and Image, San Diego, CA, 1993, pp. 45-56.

[36] P. P. Vaidyanathan, Multirate Systems and Filter Banks. Englewood Cliffs, NJ: Prentice-Hall, 1993.

[37] — , "Theory of optimal orthonormal subband coders," IEEE Trans. Signal Processing, vol. 46, pp. 1528-1543, June 1998.

[38] P. P. Vaidyanathan, Y.-P. Lin, S. Akkarakaran, and S.-M. Phoong, "Optimalilty of principal component filter banks for discrete multitone communication systems," in Proc. IEEE ISCAS'O0, Geneva, Switzerland, May 2000.

[39] - "Optimizing the capacity of orthogonal and biorthogonal DMT channels," in Proc. EUSIPCO'O0, Tampere, Finland, Sept. 2000.

[40] P. P. Vaidyanathan and S. Akkarakaran, "A review of the theory and applications of optimal subband and transform coders," J. Appl. Comput. Harmonic Analysis, to be published.

[41] L. Vandendorpe, L. Cuvelier, F. Deryck, J. Louveaux, and O. van de Wiel, "Fractionally spaced linear and decision feedback detectors for transmultiplexers," IEEE Trans. Signal Processing, vol. 46, pp. 996-1011, Apr. 1998.

[42] M. Vetterli and J. Kovačević, Wavelets and Subband Coding. Englewood Cliffs, NJ: Prentice-Hall, 1995.

[43] J.-J. Werner, "The HDSL environment," IEEE J. Select. Areas in Commun., vol. 9, pp. 785-800, Aug. 1991.

[44] X.-G. Xia, "New precoding for intersymbol interference cancellation using nonmaximally decimated multirate filter banks with ideal FIR equalizers," IEEE Trans. Signal Processing, vol. 45, pp. 2431-2441, Oct. 1997.

[45] — , "Filterbank precoders for blind equalization: Polynomial ambiguity resistant precoders (PARP)," IEEE Trans. Circuits Syst. I, vol. 48, pp. 193-209, Feb. 2001.

[46] B. Xuan and R. H. Bamberger, "FIR principal component filter banks," IEEE Trans. Signal Processing, vol. 46, pp. 930-940, Apr. 1998.

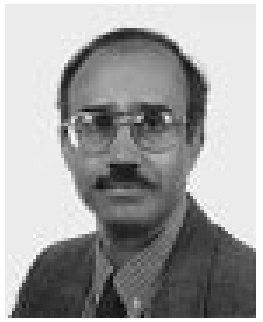

P. P. Vaidyanathan (S'80-M'83-SM'88-F'91) was born in Calcutta, India, on Oct. 16, 1954. He received the B.Sc. (Hons.) degree in physics and the B.Tech. and M.Tech. degrees in radiophysics and electronics, all from the University of Calcutta, India, in 1974, 1977, and 1979, respectively, and the Ph.D. degree in electrical and computer engineering from the University of California at Santa Barbara in 1982.

He was a Postdoctoral Fellow at the University of California, Santa Barbara from September 1982 to March 1983. In March 1983, he joined the Electrical Engineering Department of the California Institute of Technology, Pasadena, as an Assistant Professor, and since 1993, has been Professor of electrical engineering there. His main research interests are in digital signal processing, multirate systems, wavelet transforms and signal processing for digital communications. 
Dr. Vaidyanathan served as Vice-Chairman of the Technical Program committee for the 1983 IEEE International Symposium on Circuits and Systems, and as the Technical Program Chairman for the 1992 IEEE International Symposium on Circuits and Systems. He was an Associate Editor for the IEEE TRANSACTIONS ON CIRCUITS AND SySTEMS for the period 1985-1987, and is currently an Associate Editor for the journal IEEE SIGNAL PROCESSING LETTERS, and a Consulting Editor for the journal Applied and Computational Harmonic Analysis. He has been a Guest Editor in 1998 for special issues of the IEEE Transactions on Signal Processing and the IEEE Transactions ON CIRCUITS AND SYSTEMS II, on the topics of filter banks, wavelets and subband coders. He has authored a number of papers in IEEE journals, and is the author of the book Multirate Systems and Filter Banks. (NJ, Englewood Cliffs: Prentice Hall, 1993). He has written several chapters for various signal processing handbooks. He was a recipient of the Award for excellence in teaching at the California Institute of Technology for the years 1983-1984, 1992-1993, and 1993-1994. He also received the NSF's Presidential Young Investigator Award in 1986. In 1989 he received the IEEE ASSP Senior Award for his paper on multirate perfect-reconstruction filter banks. In 1990 he was recipient of the S. K. Mitra Memorial Award from the Institute of Electronics and Telecommunications Engineers, India, for his joint paper in the IETE journal. He was also the coauthor of a paper on linear-phase perfect reconstruction filter banks in the IEEE SP TRANSACTIONS, for which the first author (Truong Nguyen) received the Young Outstanding Author Award in 1993. He received the 1995 F. E. Terman Award of the American Society for Engineering Education, sponsored by Hewlett Packard Co., for his contributions to engineering education, especially the book Multirate Systems and Filter Banks. He has given several plenary talks including at the Sampta'01, Eusipco'98, SPCOM'95, and Asilomar'88 conferences on signal processing. He has been chosen a distinguished lecturer for the IEEE Signal Processing Society for the year 1996-1997. In 1999, he was chosen to receive the IEEE CAS Society's Golden Jubilee Medal. He is a recipient of the IEEE Signal Processing Society's Technical Achievement Award for the year 2002.

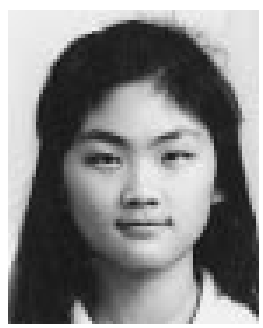

Yuan-Pei Lin (S'93-M'97) was born in Taipei, Taiwan, R.O.C., in1970. She received the B.S. degree in control engineering from the National Chiao-Tung University, Hsinchu, Taiwan, R.O.C., in 1992, and the M.S. and Ph.D. degrees, both in electrical engineering from California Institute of Technology, Pasadena, in 1993 and 1997, respectively.

She joined the Department of Electrical and Control Engineering of National Chiao-Tung University in 1997. Her research interests include multirate filter banks, wavelets and applications to communication systems. She is currently an Associate Editor for Multidimensional Systems and Signal Processing.

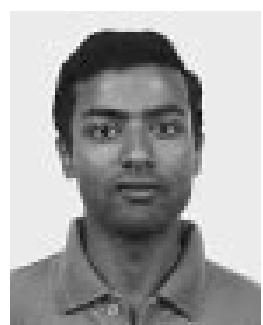

Sony Akkarakaran (S'97-M'01) was born in Thrissur, India in 1975. He received the B.Tech. degree from the Indian Institute of Technology, Bombay, in 1996, and the M.S. and the Ph.D. degrees from the California Institute of Technology, Pasadena, both in electrical engineering, in 1997 and 2001, respectively.

Since July 2001, he has been with Sequoia Communications Corp., Los Angeles, working on designing the baseband processor for a WCDMA mobile receiver. His doctoral thesis was on filter bank optimization with communications and noise suppression applications. His research interests are wireless communications, CDMA and OFDM systems, filter banks and wavelets, and digital signal processing for communications.

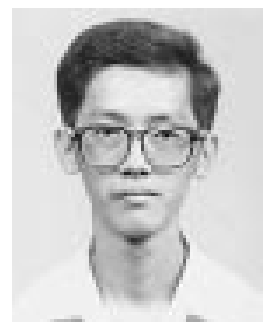

See-May Phoong (S'93-A'96) was born in Johor, Malaysia, in 1968. He received the B.S. degree in electrical engineering from the National Taiwan University, Taipei, Taiwan, in 1991, and the M.S. and $\mathrm{Ph} . \mathrm{D}$. degrees in electrical engineering from the California Institute of Technology (Caltech), Pasadena, in 1992 and 1996, respectively.

$\mathrm{He}$ joined the faculty of the Department of Electronic and Electrical Engineering, Nanyang Technological University, Singapore, from September 1996 to September 1997. In September 1997, he joined the Graduate Institute of Communication Engineering, National Taiwan University , Taipei, Taiwan, as an Assistant Professor and since 2002, he has been an Associate Professor. His interests include multirate signal processing, filter banks and their application to communications.

Dr. Phoong is currently an Associate Editor for IEEE TRANSACTIONS ON Circuits AND Systems II, and IEEE Signal Processing LeTters. He is the recipient of the Wilts Prize (1997) for outstanding independent research in Electrical Engineering at Caltech. 